\title{
ACCESSORY PARAMETERS FOR PUNCTURED SPHERES
}

\author{
IRWIN KRA
}

\begin{abstract}
This paper contains some qualitative results about the accessory parameters for punctured spheres with signature. We show that the Fuchsian uniformizing connection, and hence also the accessory parameters, for the surface depends real analytically on moduli. We also show that the important invariants of a uniformization of a punctured sphere such as the accessory parameters, Fuchsian groups, Poincaré metrics, and covering maps vary continuously under degenerations such as coalescing of punctures.
\end{abstract}

The (branched) holomorphic universal covering group of a Riemann surface $S$ of finite analytic type $(g, n)$ with $2 g-2+n>0$ can be described as the monodromy group of the Schwarzian differential equation

$$
\{f, \cdot\}=\varphi,
$$

where $\varphi$ is a meromorphic connection on $S$ with singularities of very simple type and only at the punctures of $S$. The connection $\varphi$ depends on $3 g-3+n$ unknown constants called accessory parameters. Despite numerous attempts (see Hejhal [He2] for some historical remarks), these parameters remain elusive. They can be determined only in the cases where the covering group is elementary or the surface has no moduli (see \$2.2). Nevertheless, the study of the parameters has led to some surprising results. Hejhal [He2] has used variations of accessory parameters to study Poincare series, and in the work of Zograf and Takhtadzhyan [ZT1, ZT2, ZT3] these accessory parameters appear in calculations of the potential for the Weil-Petersson metric on Teichmüller space.

In this paper we study the simplest case, that is, $n$-punctured spheres $(g=$ $0)$. In this case the connection $\varphi$ is a rational function. We show that $\varphi$ (and hence also the accessory parameters) depends real analytically on moduli and that various objects attached to a uniformization (such as the uniformizing

Received by the editors November $30,1987$.

1980 Mathematics Subject Classification (1985 Revision). Primary 30F10, 30F35, 32G15, 14H15.

Research partially supported by National Science Foundation grants 8401280 and 8120790 . 
connection, the Poincare metric, the covering group, and covering map) behave well under degeneration (coalescing points or shrinking curves).

\section{CONNECTION ASSOCiated to A UNiformization}

1.1. Let $\Gamma$ be a (usually, but not always, nonelementary) finitely generated function group with invariant component $\Delta$. We introduce some standard notation:

$\pi: \Delta \rightarrow \Delta / \Gamma=S$, a fixed canonical projection,

$\Delta_{0}=\Delta_{0 \Gamma}=$ complement in $\Delta$ of the elliptic fixed points of $\Gamma$,

$S_{0}=\pi\left(\Delta_{0}\right)=\Delta_{0} / \Gamma$,

$\bar{\Delta}=\bar{\Delta}_{\Gamma}=\Delta \cup\{$ fixed points of parabolic elements of $\Gamma$ corresponding to punctures on $S\}$,

$\bar{S}=$ compactification of $S=\bar{\Delta} / \Gamma=\pi(\bar{\Delta})$,

$E=$ exceptional set $=\bar{S}-S_{0}=\pi\left(\bar{\Delta}-\Delta_{0}\right)=\left(\bar{\Delta}-\Delta_{0}\right) / \Gamma$,

$(g, n)=$ type of $\Gamma=$ type of $\Delta_{0} / \Gamma$, and

$\left(g, n ; \nu_{1}, \ldots, \nu_{n}\right)=$ signature of $\Gamma$.

By abuse of notation we shall say that (the compact surface) $\bar{S}$ has signature $\left(g, n ; \nu_{1}, \ldots, \nu_{n}\right)$. We shall say that $\bar{S}$ has hyperbolic signature if $\Gamma$, as above, is nonelementary, that is, if and only if

$$
2 g-2+\sum_{j=1}^{n}\left(1-\nu_{j}^{-1}\right)>0 .
$$

Let $P_{0} \in S_{0}$ and let $\zeta$ be a local coordinate on $S_{0}$ defined in a neighborhood of $P_{0}$. Let $f$ be a local inverse for $\pi$ near $P_{0}$. We define a function $\varphi$ of $\zeta$ by

$$
\varphi(\zeta)=\{f, \zeta\}
$$

where $\{f, \cdot\}$ is the Schwarzian derivative of $f$, given by the formula

$$
\{f, \cdot\}=\frac{f^{\prime \prime \prime}}{f^{\prime}}-\frac{3}{2}\left(\frac{f^{\prime \prime}}{f^{\prime}}\right)^{2}=\left(\frac{f^{\prime \prime}}{f^{\prime}}\right)^{\prime}-\frac{1}{2}\left(\frac{f^{\prime \prime}}{f^{\prime}}\right)^{2} .
$$

We observe that $\varphi$ is independent of the choice of $f$, since an arbitrary local inverse for $\pi$ is of the form $\gamma \circ f$ with $\gamma \in \Gamma$ and the Cayley identity yields

$$
\{\gamma \circ f, \zeta\}=\{\gamma, f(\zeta)\} f^{\prime}(\zeta)^{2}+\{f, \zeta\}=\{f, \zeta\} .
$$

Replacing $\zeta$ by another local coordinate $\tilde{\zeta}$ at $P_{0}$, we see that

$$
\tilde{\varphi}(\tilde{\zeta})=\{f, \tilde{\zeta}\}=\{f, \zeta\}\left(\frac{d \zeta}{d \tilde{\zeta}}\right)^{2}+\{\zeta, \tilde{\zeta}\}=\varphi(\zeta)\left(\frac{d \zeta}{d \tilde{\zeta}}\right)^{2}+\{\zeta, \tilde{\zeta}\} ;
$$

thus the collection

$$
\left\{\varphi(\zeta) ; \zeta \text { is a local coordinate on } S_{0}\right\}
$$

defines a projective connection on $S_{0}$. We call it the uniformizing connection corresponding to $\pi$, and we will denote it by the symbol $\eta_{\pi}$. 
We refer the reader to Gunning [G, pp. 164-219] for a discussion of connections and to Hille [Hi, pp. 375-377] for a treatment of Schwarzian derivatives.

1.2. The group $\Gamma$ yields a projective structure on $S_{0}$. The coordinates $\zeta$ of this projective structure have the property that the local inverses $f$ of $\pi$ are given by maps $\gamma \in \Gamma$. Thus with respect to these projective coordinates, $\eta_{\pi}$ is represented by the zero function.

1.3. Let us start with an arbitrary connection $\eta$ on $S_{0}$. Let $\pi_{1}\left(S_{0}, P\right)$ be the fundamental group of $S_{0}$ based at $P \in S_{0}$. Let $\zeta$ be an arbitrary local coordinate vanishing at $P$ and let $\eta$ have the value $\varphi$ in terms of $\zeta$. Let $\psi$ be a solution of the Schwarzian differential equation

$$
\{\psi, \zeta\}=\varphi(\zeta)
$$

we normalize $\psi$ so that

$$
\psi(\zeta)=\zeta+O\left(\zeta^{3}\right), \quad \zeta \rightarrow 0 .
$$

Let $\alpha$ be a closeld path in $S_{0}$ beginning at $P$. Continuing the solution $\psi$ around $\alpha$, we get a new solution $\tilde{\psi}$. It follows that

$$
\tilde{\psi}=\gamma \circ \psi \quad \text { with } \gamma \in \operatorname{PSL}(2, \mathbf{C}) \text {. }
$$

The Möbius transformation $\gamma$ depends only on the homotopy class of the curve $\alpha$, and the map sending $\alpha$ to $\gamma$ is a group homomorphism known as the monodromy homomorphism

$$
\eta^{*}: \pi_{1}\left(S_{0}, P\right) \rightarrow \operatorname{PSL}(2, \mathrm{C}) .
$$

We note that if our solution $\psi$ of (1.3.1) is evaluated in terms of another local coordinate $\tilde{\zeta}$, then it still solves (1.3.1) but corresponds to a different normalization than (1.3.2). A different normalization of the initial solution corresponds to replacing $\psi$ by $A \circ \psi$ with $A \in \operatorname{PSL}(2, \mathbf{C})$. This involves replacing $\gamma$ by $A \circ \gamma \circ A^{-1}$. Thus the projective connection $\eta$ determines the monodromy homomorphism only up to conjugation by an element of $\operatorname{PSL}(2, \mathrm{C})$.

1.4. Let $\rho: U \rightarrow \Delta_{0}$ be a holomorphic universal covering map, where $U$ is the upper half-plane. Let $F$ be Fuchsian model of the action of $\Gamma$ on $\Delta_{0}$ and let $H$ be the covering group of $\rho$; that is, $\Delta_{0} / \Gamma \cong U / F=S_{0}, U / H \cong \Delta_{0}$, and

$$
\begin{aligned}
H & =\{h \in \operatorname{PSL}(2, \mathbf{R}) ; \rho \circ h=\rho\}, \\
F & =\{h \in \operatorname{PSL}(2, \mathbf{R}) ; \rho \circ h=\gamma \circ \rho \text { for some } \gamma \in \Gamma\} .
\end{aligned}
$$

We then have an exact sequence of groups and group homomorphisms

$$
1 \rightarrow H \rightarrow F \stackrel{\rho^{*}}{\rightarrow} \Gamma \rightarrow 1,
$$

with

$$
\rho \circ h=\rho^{*}(h) \circ \rho, \quad \text { all } h \in F .
$$

We can identify $F$ with $\pi_{1}\left(S_{0}, P\right)$; in this case $\eta_{\pi}^{*}$ becomes identified with $\rho^{*}$. 
1.5. The group $\Gamma$ also introduces distinguished coordinates at the points of $E \subset \bar{S}$. The uniformizing connection $\eta_{\pi}$ has singularities at the points of $E$. We proceed to describe these singularities. The starting point is the identity $\pi \circ f=I$ and its consequence

$$
\{\pi, f(\zeta)\} f^{\prime}(\zeta)^{2}+\varphi(\zeta)=0 .
$$

Assume that $z_{0} \in \pi^{-1}(E)$ is an elliptic fixed point of order $\nu(2 \leq \nu<\infty)$. By conjugation, we assume that $z_{0}=0$ and the stabilizer $z_{0}$ in $\Gamma$ is generated by the mapping $A(z)=e^{2 \pi i / \nu} z$. We choose a local coordinate $\zeta$ on $S$ so that in a neighborhood of $z_{0}=0, \zeta=\pi(z)=z^{\nu}$. It follows that

$$
\varphi(\zeta)=-\left\{z^{\nu}, z\right\}\left(\frac{d z}{d \zeta}\right)^{2}=\frac{\nu^{2}-1}{2} z^{-2}\left(\frac{d z}{d \zeta}\right)^{2}=\frac{1}{2}\left(1-\nu^{-2}\right) \zeta^{-2} .
$$

Similarly, if $P \in \bar{S}-S$, then by conjugation we may assume that $\Delta$ contains an upper half-plane

$$
U_{c}=\{z \in \mathbf{C} ; \operatorname{Im} z>c\}, \text { for some } c>0,
$$

that is precisely invariant under $A(z)=z+1$. We can choose a local coordinate $\zeta$ vanishing at $P$ so that $\zeta=e^{2 \pi i z}, z \in U_{c}$. Again starting from (1.5.1) we see that

$$
\varphi(\zeta)=-\left\{e^{2 \pi i z}, z\right\}\left(\frac{d z}{d \zeta}\right)^{2}=2 \pi^{2}\left(\frac{d \zeta}{d z}\right)^{2}=\frac{1}{2} \zeta^{-2} .
$$

Comparing (1.5.4) with (1.5.2), we see that the parabolic case is the limiting case as $\nu$ approaches $\infty$ of the elliptic order $\nu$ case.

We conclude that the uniformizing connection $\eta_{\pi}$ extends as a meromorphic connection to $\bar{S}$. If $P \in \bar{S}-S_{0}$ and $\zeta$ and $\tilde{\zeta}$ are two coordinates vanishing at $P$ and if $\varphi$ and $\tilde{\varphi}$ are the representatives of $\eta_{\pi}$ in terms of these coordinates, then we conclude from (1.5.2) and (1.5.4) that while the singular part of $\eta_{\pi}$ at $P$ is not well defined, the residue of $\eta_{\pi}$ at $P$ is well defined. It should be obvious to the reader that by the residue of $\eta_{\pi}$ at $P$ we mean the coefficient of $\zeta^{-2}$ for the Laurent series expansion of $\varphi$, where $\varphi$ is a representative of $\eta_{\pi}$ in terms of any local coordinate $\zeta$ that vanishes at $P$.

\section{TRIVIALIZATION OF CONNECTIONS: PUNCTURED SURFACES}

2.1. Let $\bar{S}$ be a surface of signature $\left(0, n ; \nu_{1}, \ldots, \nu_{n}\right), n \geq 3$. In this case, we can use a single global coordinate $z$ on $\bar{S}=\mathbf{C} \cup\{\infty\}=\widehat{\mathbf{C}}$ to describe the uniformizing connection $\eta_{\pi}$. We proceed to describe the representative $\varphi$ of $\eta_{\pi}$ in terms of $z$. Let

$$
S_{0}=\widehat{\mathbf{C}}-\left\{\lambda_{1}, \ldots, \lambda_{n}\right\} .
$$

We observe that if the surface $S$ is replaced by $A^{-1}(S)$ with $A \in \operatorname{PSL}(2, \mathrm{C})$, then the covering map $\pi$ is replaced by $A^{-1} \circ \pi$ and hence the representative 
for $\eta_{A^{-1} \pi}$ is $(\varphi \circ A)\left(A^{\prime}\right)^{2}$. We conclude from this or directly that if $\infty \in S_{0}$, then

$$
\varphi(z)=O\left(|z|^{-4}\right), \quad z \rightarrow \infty ;
$$

and if $\infty$ is the image of a branch point of order $\nu(2 \leq \nu \leq \infty)$, then

$$
\varphi(z)=\frac{1}{2}\left(1-\nu^{-2}\right) z^{-2}+\sum_{j=-3}^{\infty} a_{j} z^{j}, \quad|z| \text { large } .
$$

With these preliminaries out of the way, let us consider the case $n=3$. By conjugation, we assume $\lambda_{1}=0, \lambda_{2}=1, \lambda_{3}=\infty$. It follows that

$$
\varphi(z)=\frac{1}{2}\left(1-\nu_{1}^{-2}\right) z^{-2}+\frac{1}{2}\left(1-\nu_{2}^{-2}\right)(z-1)^{-2}+a z^{-1}+b(z-1)^{-1},
$$

where $a$ and $b$ are constants that guarantee the expansion (2.1.1) with $\nu=\nu_{3}$. It follows that

$$
a=-b=\frac{1}{2}\left(1-\nu_{1}^{-2}-\nu_{2}^{-2}+\nu_{3}^{-2}\right) \text {. }
$$

2.2. The above calculations prove the well-known fact that a uniformization of a thrice punctured sphere is determined uniquely (up to conjugation) by the three ramification numbers. Let us set

$$
\begin{aligned}
\Phi(z)=\frac{1}{2}\left[\left(1-\nu_{1}^{-2}\right) z^{-2}+\left(1-\nu_{2}^{-2}\right)(z-1)^{-2}\right. \\
\left.+\left(1-\nu_{1}^{-2}-\nu_{2}^{-2}+\nu_{3}^{-2}\right)(1 / z-1 /(z-1))\right] .
\end{aligned}
$$

We observe for future use that if $A$ is a Möbius transformation that permutes $0,1, \infty$ and preserves ramification numbers (for example, if $\nu_{1}=\nu_{2}=\nu_{3}$ ), then

$$
\Phi=(\Phi \circ A)\left(A^{\prime}\right)^{2} \text {. }
$$

2.3. We return to the general case $n \geq 4$. As before, we normalize so that $\lambda_{1}=0, \lambda_{2}=1, \lambda_{3}=\infty$. We have some freedom in choosing a basis for the cusp forms on $S_{0}$ (these are allowed to have simple poles at the points in $\left.E=\left\{\lambda_{1}, \ldots, \lambda_{n}\right\}\right)$. We will write the uniformizing connection in the form

$$
\varphi(z)=\Phi(z)+\sum_{j=4}^{n} \frac{\lambda_{j}\left(\lambda_{j}-1\right)}{z(z-1)\left(z-\lambda_{j}\right)}\left[\frac{1}{2}\left(1-\nu_{j}^{-2}\right) \frac{1}{z-\lambda_{j}}+c_{j}\right],
$$

where $c_{4}, \ldots, c_{n}$ are $n-3$ constants that depend on the uniformization and are known as the accessory parameters. It is useful to think of $\varphi(z) d z^{2}$ as a holomorphic quadratic differential on $S_{0}$ with double poles at the points of $E$ (with prescribed residues). Since

$$
z(z-1) / d z
$$

is a holomorphic reciprocal differential on $\widehat{\mathbf{C}}$, it follows that

$$
c_{j}=\frac{1}{\lambda_{j}\left(\lambda_{j}-1\right)} \operatorname{Res}_{\lambda_{j}} z(z-1) \varphi(z) d z, \quad j=4, \ldots, n .
$$


2.4. It is useful, at times, to examine the connection $\eta_{\pi}$ on $\Delta$. Let $\psi=$ $\{\pi, \cdot\}$. Then $\psi$ is an automorphic form on $\bar{\Delta}$, regular on $\bar{\Delta}-\pi^{-1}(E)$, and

$$
\psi(z)=-\varphi(\pi z) \pi^{\prime}(z)^{2}, \quad z \in \Delta .
$$

Using (2.4.1) or the definition of $\psi$, we can describe the singularities of $\psi$.

If $z_{0} \in \Delta$ is a fixed point of order $\nu, 2 \leq \nu<\infty$, then

$$
\psi(z)=\frac{1}{2}\left(1-\nu^{2}\right)\left(z-z_{0}\right)^{-2}+O(1), \quad z \rightarrow z_{0} .
$$

Without loss of generality we can assume, by conjugating $\Gamma$ if necessary, that $z_{0}=0, \pi\left(z_{0}\right)=0$, and that the stabilizer of $z_{0}$ in $\Gamma$ is generated by $A(z)=$ $e^{2 \pi i / \nu} z$. Thus we have

$$
\pi(z)=\sum_{j=1}^{\infty} a_{j} z^{\nu j}, \quad a_{1} \neq 0, \text { for }|z| \text { small } .
$$

We can write

$$
\pi(z)=h\left(z^{\nu}\right), \quad h(z)=\sum_{j=1}^{\infty} a_{j} z^{j} .
$$

It follows that

$$
\begin{aligned}
\psi(z) & =\{\pi, z\}=\left\{h, z^{\nu}\right\}\left(\nu z^{\nu-1}\right)^{2}+\left\{z^{\nu}, z\right\} \\
& =\frac{1}{2}\left(1-\nu^{2}\right) z^{-2}+\text { (holomorphic function). }
\end{aligned}
$$

To compute $\varphi$, note that $f=\pi^{-1}$ has the form

$$
f(z)=b_{0} z^{1 / \nu}\left(1+b_{1} z+b_{2} z^{2}+\cdots\right)=b_{0} g(z)^{1 / \nu},
$$

where

$$
g(z)=z+\nu b_{1} z^{2}+\cdots, \quad|z| \text { small } .
$$

From $\pi \circ f=I$, we see that

$$
b_{0}=a_{1}^{-1 / \nu}, \quad b_{1}=-\nu^{-1} a_{2} a_{1}^{-2} .
$$

It follows that (with $G(z)=z^{1 / \nu}$ )

$$
\begin{aligned}
\varphi(z) & =\{G, g(z)\} g^{\prime}(z)^{2}+\{g, z\} \\
& =\frac{1}{2}\left(1-\nu^{-2}\right) g(z)^{-2} g^{\prime}(z)^{2}+\{g, z\} \\
& =\frac{1}{2}\left(1-\nu^{-2}\right)\left(z^{-2}-2 a_{2} a_{1}^{-2} z^{-1}\right)+O(1), \quad z \rightarrow 0 .
\end{aligned}
$$

For the parabolic case, we normalize so that $\Delta$ contains the half-plane $U_{c}$ defined by (1.5.3) that is precisely invariant under the subgroup of $\Gamma$ generated by $A(z)=z+1$. It is easy to see that

$$
\psi(z)=2 \pi^{2}+\sum_{m=2}^{\infty} b_{m} e^{2 \pi i m z}, \quad z \in U_{c},
$$


and if $\lambda=\pi(\infty) \in \mathbf{C}$, then

$$
\pi(z)=\sum_{m=0}^{\infty} a_{m} e^{2 \pi i m z}, \quad z \in U_{c}, a_{1} \neq 0 .
$$

It follows from the above formulae that

$$
\varphi(z)=\frac{1}{2}(z-\lambda)^{-2}-a_{2} a_{1}^{-2}(z-\lambda)^{-1}+O(1), \quad z \rightarrow \lambda .
$$

The easiest way to verify (2.4.2) is to consider the following commutative diagram (without loss of generality $\lambda=0$ )

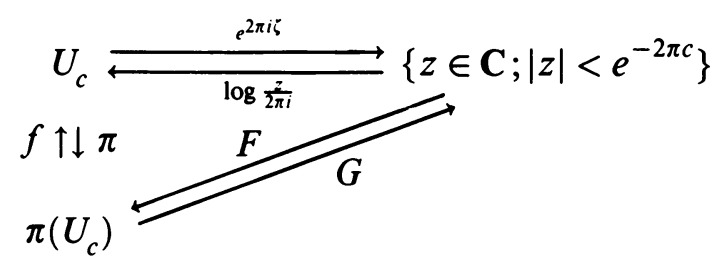

where

$$
F(z)=\sum_{m=1}^{\infty} a_{m} z^{m}, \quad|z|<e^{-2 \pi c}
$$

and

$$
G=F^{-1}, \quad f=\pi^{-1}
$$

Now

$$
\varphi(z)=\{f, z\}=\left\{\log \frac{G}{2 \pi i}, z\right\}=\frac{1}{2} G(z)^{-2} G^{\prime}(z)^{2}+\{G, z\} .
$$

Since $\{G, \cdot\}$ is holomorphic in a neighborhood of the origin, we need only compute the Laurent series expansion of $G^{\prime} / G$ at zero. This calculation is striaghtforward.

Alternately, the accessory parameter can be computed using (2.3.2).

2.5. Given $\bar{S}$ (with its signature), there are many groups $\Gamma$ that uniformize $\bar{S}$. Each group determines several connections.

A given function group $\Gamma$ with invariant component $\Delta$ (except for quasiFuchsian $\Gamma, \Delta$ is determined by $\Gamma$ ) determines only the conformal equivalence class of $\bar{S}$. The covering map $\pi$ of $S$ is determined up to composition on the left by a Möbius transformation $A^{-1}$ and composition on the right by a conformal self-map $g^{-1}$ of $\Delta$ that normalizes the group $\Gamma$. The map $g$ need not be a Möbius transformation. Thus the uniformizing connection $\varphi=\{\pi, \cdot\}$ may, in general, be replaced by

$$
\{g, f \circ A\}\left((f \circ A)^{\prime}\right)^{2}+(\varphi \circ A)\left(A^{\prime}\right)^{2} \quad(f \text { is an inverse to } \pi) .
$$


Despite the above ambiguities, there are cases where $\bar{S}$ determines uniquely the uniformizing connection-for example, if we insist on a Fuchsian uniformization (see $\S 4)$.

\section{TRIVIALIZATION OF CONNECTIONS: INTERMEDIATE PLANAR COVERINGS}

3.1. Let us consider two function groups $\Gamma$ and $G$ acting discontinuously on domains $\Delta$ and $D$ (these need not be the full invariant components). Assume we are given a covering map $f: \Delta \rightarrow D$ that induces a surjective homomorphism $f^{*}: \Gamma \rightarrow G$ with

$$
f \circ \gamma=f^{*}(\gamma) \circ f, \quad \text { all } \gamma \in \Gamma \text {. }
$$

The map $f$ induces a conformal map $F: \Delta / \Gamma \rightarrow D / G$ such that

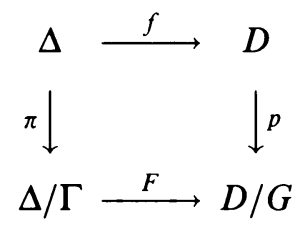

commutes, where $\pi$ and $p$ are the natural projections. We are interested in comparing the two uniformizing connections: $\eta_{\pi}$ and $\eta_{p}$. Let $\Delta_{0}$ and $D_{0}$ be the complements in $\Delta$ and $D$ of the elliptic fixed points of $\Gamma$ and $G$, respectively. The groups $\Gamma$ and $G$ yield distinguished local coordinates $\zeta$ and $z$ on $\Delta_{0} / \Gamma$ and $D_{0} / G$ such that $\eta_{\pi}$ and $\eta_{p}$ are represented by the zero functions with respect to these coordinates. We compute next the representative of $\eta_{\pi}$ with respect to the local coordinate $z$ pulled back to $\Delta / \Gamma$ via $F$ :

$$
\begin{aligned}
\varphi(z)= & \left\{\pi^{-1} \circ F^{-1}, z\right\}=\left\{f^{-1} \circ p^{-1}, z\right\}=\left\{f^{-1}, p^{-1}(z)\right\}\left(p^{-1}\right)^{\prime}(z)^{2} \\
& +\left\{p^{-1}, z\right\}=\left\{f^{-1}, z\right\} .
\end{aligned}
$$

Thus the global function $\varphi$ on $D$ defined by (3.1.1) trivializes the connection $\eta_{\pi}$ with respect to $z$, whenever two different branches of $f^{-1}$ differ by a Möbius transformation (for example, if $f$ is injective or $\Delta$ is a disk). We note that in this case $\varphi$ is a holomorphic automorphic 2-form for $G$ on $D$. For, given $g \in G, z \in D$, we have

$$
\begin{aligned}
\varphi(g z) g^{\prime}(z)^{2} & =\left\{f^{-1}, g(z)\right\} g^{\prime}(z)^{2}=\left\{f^{-1} \circ g, z\right\}=\left\{\gamma \circ f^{-1}, z\right\} \\
& =\left\{f^{-1}, z\right\}=\varphi(z)
\end{aligned}
$$

where $\gamma \in \Gamma$ is chosen so that $f^{*}(\gamma)=g$.

The nature of the cover $f$ may yield a description of certain singularities (not in $D$, of course) of $\varphi$.

3.2. We shall consider a special case. Let $\omega_{1}$ and $\omega_{2}$ be two complex numbers linearly independent over $\mathbf{R}$. Form

$$
\Omega=\left\{z \in \mathbf{C} ; z \neq n_{1} \omega_{1}+n_{2} \omega_{2} \text {, all } n_{1}, n_{2} \in \mathbf{Z}\right\} .
$$


Let $U$ be the upper half-plane, and

$$
\rho: U \rightarrow \Omega
$$

a holomorphic universal covering map. The group $G$ generated by

$$
A(z)=z+\omega_{1}, \quad B(z)=z+\omega_{2},
$$

acts on $\Omega$, and $\Omega / G$ is a surface of type $(1,1)$. Similarly, the group $\widetilde{G}$ generated by $G$ and $C(z)=-z$ acts on $\Omega$ and $\Omega / \widetilde{G}$ has signature $(0,4 ; \infty, 2,2,2)$.

Let $\mathscr{P}=\mathscr{P}(\Omega, \cdot)$ be the Weierstrass $\mathscr{P}$-function for $\Omega$; that is, for $z \in \mathbf{C}$, (3.2.1)

$$
\mathscr{P}(\Omega, z)=z^{-2}+\sum_{\left(n_{1}, n_{2}\right) \in \mathbf{Z} \oplus \mathbf{Z}-\{0\}}\left[\left(z-n_{1} \omega_{1}-n_{2} \omega_{2}\right)^{-2}-\left(n_{1} \omega_{1}+n_{2} \omega_{2}\right)^{-2}\right] .
$$

Then $\mathscr{P}$ induces a holomorphic map from $\Omega / G$ to $\Omega / \widetilde{G} \cong \widehat{\mathbf{C}}$ that is branched over $\infty$ and the three points $e_{1}, e_{2}, e_{3}$, where

$$
e_{1}=\mathscr{P}\left(\frac{\omega_{1}}{2}\right), \quad e_{2}=\mathscr{P}\left(\frac{\omega_{2}}{2}\right), \quad e_{3}=\mathscr{P}\left(\frac{\omega_{1}+\omega_{2}}{2}\right) .
$$

Let $\Gamma$ and $\tilde{\Gamma}$ be the Fuchsian models of the action of $G$ and $\widetilde{G}$, respectively, on $\Omega$. Then $\Gamma$ has signature $(1,1 ; \infty)$ and $\widetilde{\Gamma}$ has signature $(0,4 ; \infty, 2,2,2)$. Let $\pi$ and $\tilde{\pi}$ be the canonical coverings

$$
\pi: U \rightarrow U / \Gamma, \quad \tilde{\pi}: U \rightarrow U / \tilde{\Gamma} .
$$

By the results of $\S 3.1$, the uniformizing connections $\eta_{\pi}$ and $\eta_{\tilde{\pi}}$ are trivialized on $\Omega$ by the function $\left\{\rho^{-1}, \cdot\right\}$. Since $\rho$ is defined uniquely up to composition on the right by a Möbius transformation, it follows that this trivialization is independent of the choice of covering map $\rho$. The calculations of $\S 1.5$ show that the trivializations of these uniformizing connections on $\Omega$ are given by

$$
\psi(z)=\left\{\rho^{-1}, z\right\}=\frac{1}{2} \mathscr{P}(\Omega, z)+c(\Omega),
$$

where $c(\Omega)$ is the accessory parameter for the punctured torus $\Omega / \Gamma$ (studied extensively by Keen; see, for example, [KRV]).

From the obvious identity (valid for $\lambda \neq 0$ )

$$
\lambda^{2} \mathscr{P}(\lambda \Omega, \lambda z)=\mathscr{P}(\Omega, z)
$$

and the fact that $\lambda \rho$ covers $\lambda \Omega$, we see that

$$
\frac{1}{2} \mathscr{P}(\lambda \Omega, z)+c(\lambda \Omega)=\frac{1}{2 \lambda^{2}} \mathscr{P}\left(\Omega, \frac{z}{\lambda}\right)+\frac{1}{\lambda^{2}} c(\Omega),
$$

and hence

$$
c(\Omega)=\lambda^{2} c(\lambda \Omega) .
$$

We want to study $c(\tau)=c\left(\Omega_{\tau}\right)$, where $\boldsymbol{\Omega}_{\tau}=\{z \in \mathbf{C} ; z \neq n+m \tau$, all $n, m \in \mathbf{Z}\}$, with $\tau \in \mathbf{C}$ and $\operatorname{Im} \tau>0$. If $\tau^{\prime}=(a \tau+b) /(e \tau+d), a, b, e, d \in \mathbf{Z}$, $a d-b e=1$, then

$$
\Omega_{\tau}=\lambda \Omega_{\tau^{\prime}},
$$


where $\lambda=e \tau+d$. It follows that

$$
c(\tau)=(e \tau+d)^{-2} c((a \tau+b) /(e \tau+d)) .
$$

3.3. Let $\varphi$ be the trivialization of $\eta_{\tilde{\pi}}$ on $U / \widetilde{\Gamma}$. Since

$$
\tilde{\pi}=\mathscr{P} \circ \rho,
$$

it follows that

$$
\varphi(z)=\left\{\tilde{\pi}^{-1}, z\right\}=\left\{\rho^{-1}, \mathscr{P}^{-1}(z)\right\}\left(\mathscr{P}^{-1}\right)^{\prime}(z)^{2}+\left\{\mathscr{P}^{-1}, z\right\} .
$$

We have seen that

$$
\left\{\rho^{-1}, \zeta\right\}=\frac{1}{2} \mathscr{P}(\zeta)+c(\Omega)=\psi(\zeta),
$$

and we now observe that

$$
\beta(z)=\left\{\rho^{-1}, \mathscr{P}^{-1}(z)\right\}\left(\mathscr{P}^{-1}\right)^{\prime}(z)^{2}, \quad z \in \mathbf{C},
$$

is the projection to $\mathrm{C}-\left\{e_{1}, e_{2}, e_{3}\right\}$ of the quadratic differential $\frac{1}{2} \mathscr{P}(\zeta)+c(\Omega)$ on $\Omega$. Now equation (3.3.2) can be written

$$
\beta(\mathscr{P}(\zeta)) \mathscr{P}^{\prime}(\zeta)^{2}=\left\{\rho^{-1}, \zeta\right\}, \quad \zeta \in \Omega .
$$

Since

$$
\mathscr{P}^{\prime}(\zeta)^{2}=4\left(\mathscr{P}(\zeta)-e_{1}\right)\left(\mathscr{P}(\zeta)-e_{2}\right)\left(\mathscr{P}(\zeta)-e_{3}\right), \quad \zeta \in \Omega,
$$

it follows from (3.3.3) that

$$
\beta(z)=\frac{1}{8} \frac{z+2 c(\Omega)}{\left(z-e_{1}\right)\left(z-e_{2}\right)\left(z-e_{3}\right)}, \quad z \in \mathbf{C} \cup\{\infty\} .
$$

To compute $\varphi$, we must examine $\left\{\mathscr{P}^{-1}, z\right\}$. This will be done in $\S \S 3.4$ and 3.5 .

3.4. It is convenient (see, for example, [G, pp. 164-166]) to introduce two differential operators (on meromorphic functions)

$$
\begin{aligned}
& \theta_{1} f=f^{\prime \prime} / f^{\prime} \\
& \theta_{2} f=\left(f^{\prime \prime} / f^{\prime}\right)^{\prime}-\frac{1}{2}\left(f^{\prime \prime} / f^{\prime}\right)^{2}=\{f, \cdot\}=\left(\theta_{1} f\right)^{\prime}-\frac{1}{2}\left(\theta_{1} f\right)^{2} .
\end{aligned}
$$

The differential operator $\theta_{1}$ plays the same role for affine transformations $\left(\left\{A \in \operatorname{PSL}(2, \mathbf{C}) ; A=\left(\begin{array}{cc}a & b \\ 0 & a^{-1}\end{array}\right)\right\}\right)$ that $\theta_{2} \quad$ (= Schwarzian derivative) plays for projective transformations. Now the $\mathscr{P}$-function maps $\mathrm{C}$ onto $\mathrm{C} / \widetilde{G}=S$. In this case $\bar{S}=\widehat{\mathbf{C}}$ and $S_{0}=\mathbf{C}-\left\{e_{1}, e_{2}, e_{3}\right\}$. Since $\widetilde{G}$ is an affine group, we may proceed to compute, as in $\S \S 2.1-2.3, \theta_{1} f$, where $f=\mathscr{P}^{-1}$. Again, $\theta_{1} f$ is regular on $\mathrm{C}-\left\{e_{1}, e_{2}, e_{3}\right\}$. For

$$
\begin{aligned}
& h(z)=z^{\nu} \quad\left(\nu \in \mathbf{Q}^{*}\right), \\
& \left(\theta_{1} h\right)(z)=(\nu-1)(1 / z) .
\end{aligned}
$$

From these observations one concludes at once that

$$
\left(\theta_{1} f\right)(z)=-\frac{1}{2}\left[\left(z-e_{1}\right)^{-1}+\left(z-e_{2}\right)^{-1}+\left(z-e_{3}\right)^{-1}\right], \quad z \in \widehat{\mathbf{C}} .
$$


Note that

$$
\left(\theta_{1} f\right)(z)=-\frac{3}{2} z^{-1}+O(1), \quad z \rightarrow \infty
$$

as is required. It follows that

$$
\begin{aligned}
\Phi_{0}(z)= & \left(\theta_{2} f\right)(z)=\left(\theta_{1} f\right)^{\prime}(z)-\frac{1}{2}\left(\theta_{1} f\right)^{2} \\
= & \frac{3}{8}\left[\left(z-e_{1}\right)^{-2}+\left(z-e_{2}\right)^{-2}+\left(z-e_{3}\right)^{-2}\right] \\
& \quad-\frac{1}{4}\left[\left(z-e_{1}\right)^{-1}\left(z-e_{2}\right)^{-1}+\left(z-e_{1}\right)^{-1}\left(z-e_{3}\right)^{-1}\right. \\
& \left.\quad+\left(z-e_{2}\right)^{-1}\left(z-e_{3}\right)^{-1}\right], \quad z \in \widehat{\mathbf{C}} .
\end{aligned}
$$

The above function is the trivialization of the uniformizing connection of the surface of type $(0,4 ; 2,2,2,2)$ branched over $e_{1}, e_{2}, e_{3}, \infty$.

Using formula (2.3.1), formula (2.3.2), and normalizing so that $e_{1}=0$, $e_{2}=1, e_{3}=\lambda$, we obtain

$$
\Phi_{0}(z)=\frac{3}{8} \frac{z^{2}-z+1}{z^{2}(z-1)^{2}}+\frac{\lambda(\lambda-1)}{z(z-1)(z-\lambda)}\left[\frac{3}{8} \frac{1}{z-\lambda}+\frac{1}{8} \frac{2 \lambda-1}{\lambda(\lambda-1)}\right] .
$$

Remarks. (1) According to our conventions, the accessory parameter for the surface of signature $(0,4 ; 2,2,2,2)$ branched over $0,1, \infty, \lambda$ is

$$
\frac{1}{8}(2 \lambda-1) / \lambda(\lambda-1) \text {. }
$$

As remarked earlier, the accessory parameter depends on a choice of basis for the cusp forms. It is very sensitive to the choice of basis. For example, with a different (obvious) basis for cusp forms, the accessory parameter becomes $2 \lambda-1$, which is a biholomorphic function of $\lambda$ (instead of a two-to-one function of $\lambda$, as in our case).

(2) If we let $\lambda$ approach $\infty$, then the (trivialization) of the uniformizing connection converges to

$$
\frac{3}{8}\left[z^{-2}+(z-1)^{-2}\right]-\frac{1}{4}(1 / z(z-1)),
$$

which is the uniformizing connection for the surface of signature $(0,3 ; 2,2, \infty)$ branched over $0,1, \infty$. Similar results are valid for $\lambda$ approaching 0 or 1 .

3.5. From $\S \S 3.3$ and 3.4 , we conclude that the determination of the accessory parameters for tori of signature $(1,1 ; \infty)$ is equivalent to the determination of the accessory parameters for four times punctured spheres of signature $(0,4 ; 2,2,2, \infty)$.

3.6. Let $G$ be a Schottky group of genus $g \geq 2$. Let $\Omega$ be the region of discontinuity of $G$ and let $\rho: U \rightarrow \Omega$ be a holomorphic universal covering of $\Omega$ by $U$, the upper half-plane. Let $\Gamma$ be the Fuchsian model for the action of $G$ on $\Omega$. Then $\left\{\rho^{-1}, \cdot\right\}=\varphi$ defines a holomorphic 2-form for $G$ that is 
independent of the choice of $\rho$ and determines the Fuchsian cover of $\Omega / G$. It is an open problem to construct $\varphi$ in terms of invariants for the group $G$.

\section{FUCHSIAN ACCESSORY PARAMETERS FOR PUNCTURED SPHERES}

4.1. Consider the sphere $\widehat{\mathbf{C}}=\bar{S}$ of signature $\left(0, n+3 ; \nu_{1}, \ldots, \nu_{n+3}\right), n \geq$ 1. Assume that we have normalized $\bar{S}$ so that

$$
S_{0}=\left(S_{\lambda}\right)_{0}=\widehat{\mathbf{C}}-\left\{\lambda_{1}, \ldots, \lambda_{n}, 0,1, \infty\right\} .
$$

Assume further that

$$
\sum_{j=1}^{n+3}\left(1-\nu_{j}^{-1}\right)>2
$$

so that $\bar{S}$ can be uniformized by a Fuchsian group $\Gamma$; that is, we can choose a Fuchsian group $\Gamma$ acting on the upper half-plane $U$ so that $S=U / \Gamma$ (recall the notation introduced in $\S 1$ ). Let, as before, $\pi: U \rightarrow S$ be a branched holomorphic covering map (corresponding to our given signature). Since $\pi$ is unique up to composition on the right by an element of $\operatorname{PSL}(2, \mathbf{R})$, the uniformizing connection $\varphi$ of $\pi$ depends only on $\bar{S}$ (that is, (for fixed signature) on the points $\left.\lambda_{1}, \ldots, \lambda_{n}\right)$. It is hence given by

$$
\varphi(z)=\Phi(z)+\sum_{j=1}^{n} \frac{\lambda_{j}\left(\lambda_{j}-1\right)}{z(z-1)\left(z-\lambda_{j}\right)}\left[\frac{1}{2}\left(1-\nu_{j}^{-2}\right) \frac{1}{z-\lambda_{j}}+c_{j}\right],
$$

where $\Phi$ is the uniformizing connection of the sphere ramified at $0,1, \infty$ and signature $\left(0,3 ; \nu_{n+1}, \nu_{n+2}, \nu_{n+3}\right)$. We let

$$
c=\left(c_{1}, \ldots, c_{n}\right)=\left(c_{1}(\lambda), \ldots, c_{n}(\lambda)\right), \quad \lambda=\left(\lambda_{1}, \ldots, \lambda_{n}\right) .
$$

We now define

$$
W^{n}=\left\{\lambda \in \mathbf{C}^{n} ; \lambda=\left(\lambda_{1}, \ldots, \lambda_{n}\right) \text { with } \lambda_{j} \neq 0, \lambda_{j} \neq 1 \text { and } \lambda_{j} \neq \lambda_{k}, j \neq k\right\} \text {. }
$$

Our first aim is to establish the

Proposition. The map sending $\lambda$ to $c$ is real (but not complex) analytic.

Remark. The Fuchsian uniformizing connection depends only on $\bar{S}$ (it is independent of the choice of disk $U$ on which the Fuchsian group $\Gamma$ acts). It will be convenient to replace the upper half-plane by other disks as the branched holomorphic universal cover of $S$.

4.2. We start by describing two well-known models for $T(0, n+3)$, the Teichmüller space for surfaces of type $(0, n+3)$.

Let $M\left(S_{0}\right)$ be the space of Beltrami coefficients for the surface $S_{0}$. For $\mu \in M\left(S_{0}\right)$, let $w^{\mu}$ be the unique normalized (fixing $\left.0,1, \infty\right) \mu$-conformal automorphism of $\widehat{\mathbf{C}}$. Two Beltrami coefficients $\mu$ and $\nu$ are equivalent if there exists a conformal $h: w^{\mu}\left(S_{0}\right) \rightarrow w^{\nu}\left(S_{0}\right)$ so that $\left(w^{\nu}\right)^{-1} \circ h \circ w^{\mu}$ is 
homotopic to the identity on $S_{0}$. The Teichmüller space $T\left(S_{0}\right)$ is the set of equivalence classes $[\mu]$ of elements $\mu \in M\left(S_{0}\right)$; it is a model for $T(0, n+3)$.

The Teichmüller space $T(0, n+3)$ is the holomorphic universal cover of $W^{n}$. The covering map

$$
p: T(0, n+3) \rightarrow W^{n}
$$

is defined by

$$
p([\mu])=\left(w^{\mu}\left(\lambda_{1}\right), \ldots, w^{\mu}\left(\lambda_{n}\right)\right), \quad \mu \in M\left(S_{0}\right) .
$$

To describe the second model of $T(0, n+3)$, we start with $M(\Gamma)$, the set of Beltrami coefficients for $\Gamma$ (defined on $U$ ), that is,

$$
M(\Gamma)=\left\{\mu \in L^{\infty}(U) ;\|\mu\|_{\infty}<1 \text { and }(\mu \circ \gamma) \overline{\gamma^{\prime}} / \gamma^{\prime}=\mu \text {, all } \gamma \in \Gamma\right\} .
$$

We let $w_{\mu}$ be the unique normalized $\mu$-conformal self-map of $U$. Two elements $\mu$ and $\nu \in M(\Gamma)$ are equivalent provided $w_{\mu}\left|\mathbf{R}=w_{\nu}\right| \mathbf{R}$. The set of equivalence classes $T(\Gamma)$ is the second model for $T(0, n+3)$. The equivalence class of $\mu \in M(\Gamma)$ will be denoted by $[\mu]$, as before.

Each $\mu \in M(\Gamma)$ is the lift via $\pi$ of a unique $\nu \in M\left(S_{0}\right)$ with

$$
\mu(z)=\nu(\pi(z)) \overline{\pi^{\prime}(z)} / \pi^{\prime}(z), \quad z \in U .
$$

The correspondence $\mu \leftrightarrow \nu$ defines a complex analytic equivalence between $T(\Gamma)$ and $T\left(S_{0}\right)$.

Let (as before) $\bar{U}_{\Gamma}$ be the union of $U$ and the set of fixed points of parabolic elements of $\Gamma$. Let $\mu \in M(\Gamma)$ and let $\nu$ be given by (4.2.3). Let $\Gamma_{\mu}=$ $w_{\mu} \Gamma\left(w_{\mu}\right)^{-1}$. The map $\pi$ extends continuously on $\bar{U}_{\Gamma}$ and setting

$$
\pi_{\mu}=w^{\nu} \circ \pi \circ\left(w_{\mu}\right)^{-1}
$$

we obtain the commutative diagram:

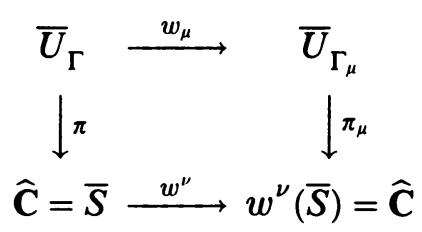

Select points $z_{j} \in \bar{U}_{\Gamma}$ so that $\pi\left(z_{j}\right)=\lambda_{j}, j=1, \ldots, n+3$ (the points $z_{j}$ are elliptic or parabolic fixed points, and without loss of generality $z_{n+1}=0$, $z_{n+2}=1$, and $z_{n+3}=\infty$ ). The map $p$ of (4.2.1) and (4.2.2) can now be identified with

$$
p([\mu])=\left(\pi_{\mu} \circ w_{\mu}\left(z_{1}\right), \ldots, \pi_{\mu} \circ w_{\mu}\left(z_{n}\right)\right), \quad \mu \in M(\Gamma) .
$$

We introduce the fiber space

$$
\tilde{T}(\Gamma)=\left\{([\mu], z) \in T(\Gamma) \times \widehat{\mathbf{C}} ; \mu \in M(\Gamma), z \in w_{\mu}\left(\bar{U}_{\Gamma}\right)=\bar{U}_{\Gamma_{\mu}}\right\}
$$


and observe that it contains $T(\Gamma) \times U$ as a dense open subspace. We define a map

$$
\pi: \widetilde{T}(\Gamma) \rightarrow T(\Gamma) \times \widehat{\mathbf{C}}
$$

by

$$
\pi([\mu], z)=\left([\mu], \pi_{\mu}(z)\right), \quad \mu \in M(\Gamma), z \in \bar{U}_{\Gamma_{\mu}} .
$$

We claim that $\pi$ is continuous, and its restriction to $T(\Gamma) \times U$ is real analytic. The real analyticity follows from [E, Lemma 4.2] and [EK, §5.3]. Continuity of $\pi$ follows from the continuity of $\pi_{\mu}$ for fixed $\mu$. We define

$$
\widetilde{T}_{0}(\Gamma)=\left\{([\mu], z) \in \widetilde{T}(\Gamma) ; \mu \in M(\Gamma), z \in w_{\mu}\left(U_{0 \Gamma}\right)\right\},
$$

where $U_{0 \Gamma}=\{z \in U ; z$ is not a fixed point of an elliptic element of $\Gamma\}$. Then it follows that $\pi \mid \widetilde{T}_{0}(\Gamma)$ is a local diffeomorphism and a covering map onto its image. The covering group is $\Gamma$ acting on $\widetilde{T}(\Gamma)$ by

$$
\gamma([\mu], z)=\left([\mu], \gamma_{\mu} z\right),
$$

where $\gamma \in \Gamma, \mu \in M(\Gamma), z \in \bar{U}_{\Gamma_{\mu}}, \gamma_{\mu}=w_{\mu} \circ \gamma \circ w_{\mu}^{-1}$. The action of $\Gamma$ on $\widetilde{T}(\Gamma)$ is real analytic and discontinuous; its action on $\widetilde{T}_{0}(\Gamma)$ is fixed point free.

4.3. We are now ready to prove Proposition 4.1. We consider the fiber space $\widetilde{W}^{n}$ over $W^{n}$ whose fiber over $\lambda \in W^{n}$ is $S_{0}$ given by (4.1.1); that is,

$$
\widetilde{W}^{n}=\left\{(\lambda, z) \in W^{n} \times \mathbf{C} ; z \neq 0,1, \lambda_{j}, j=1, \ldots, n\right\}=W^{n+1} .
$$

We claim that $\pi \mid \widetilde{T}_{0}(\Gamma)$ induces a map

$$
\tilde{\pi}: \widetilde{T}_{0}(\Gamma) \rightarrow \widetilde{W}^{n}
$$

defined by

$$
\tilde{\pi}([\mu], z)=\left(p([\nu]), \pi_{\mu}(z)\right),
$$

where $\mu \in M(\Gamma), z \in U_{0 \Gamma_{\mu}}$, and $\nu$ is defined by (4.2.3). The map $\tilde{\pi}$ is a real-analytic local diffeomorphism. Let $\zeta_{0}=\left(\lambda_{0}, z_{0}\right) \in \widetilde{W}^{n}$ and let $\rho$ be a local inverse to $\tilde{\pi}$ defined in a neighborhood of $\zeta_{0}$. Write

$$
\rho(\lambda, z)=(\delta(\lambda), f(\lambda, z)),
$$

where $\delta(\lambda) \in T(\Gamma), f(\lambda, z) \in U$. Then $f(\lambda, z)$ is real analytic in $(\lambda, z)$ and, for fixed $\lambda$, complex analytic in $z$. So is

$$
\varphi(\lambda, z)=\{f(\lambda, z), z\} .
$$

Furthermore, we know that $\varphi(\lambda, z)$ is a rational function of $z$ (for fixed $\lambda$ ). We conclude that the accessory parameters depend real analytically on $\lambda$. Note that $\varphi$ is a globally defined real-analytic function on $\widetilde{W}^{n}$.

We claim that $\varphi$ is not a complex analytic function on $\widetilde{W}^{n}$ (alternately, $c$ is not a complex analytic function on $\left.W^{n}\right)$. Let $\lambda^{0} \in W^{n}$ and assume that $c(\lambda)$ is complex analytic in some neighborhood of $\lambda^{0}$. Choose a point $b \in\left(S_{\lambda^{0}}\right)_{0}$ 
and closed curves $\alpha_{1}, \ldots, \alpha_{n+2}$ through $b$ whose homotopy classes generate $\pi_{1}\left(\left(S_{\lambda^{0}}\right)_{0}, b\right)$. Then these curves also generate $\pi_{1}\left(\left(S_{\lambda}\right)_{0}, b\right)$ for all $\lambda$ sufficiently close to $\lambda^{0}$. Let $D$ be a simply connected neighborhood of $b$ in $S_{\lambda^{0}}$. Then $D$ is also contained in $S_{\lambda}$ for all $\lambda$ sufficiently close to $\lambda^{0}$. For all such $\lambda$, solve the equation

$$
\begin{gathered}
\left\{f_{\lambda}, z\right\}=\varphi(\lambda, z), \quad z \in D, \\
f_{\lambda}(b)=0, \quad f_{\lambda}^{\prime}(b)=1, \quad f_{\lambda}^{\prime \prime}(b)=0 .
\end{gathered}
$$

Then $f_{\lambda}$ depends holomorphically on $\lambda$. Continuation of $f_{\lambda}$ along $\alpha_{j}, j=$ $1, \ldots, n+2$, leads to $\gamma_{j}^{\lambda} \circ f_{\lambda}$, with $\gamma_{j}^{\lambda} \in \operatorname{PSL}(2, \mathbf{C})$. The motions $\left\{\gamma_{1}^{\lambda}, \ldots, \gamma_{n+2}^{\lambda}\right\}$ generate a Fuchsian group. Let us choose a simple path $\alpha$ in $\left(S_{\lambda^{0}}\right)_{0}$ that is neither contractible to a point nor contractable to a puncture. Then $\gamma^{\lambda}$, the motion resulting from continuing $f_{\lambda}$ around $\alpha$, must be hyperbolic and the function that sends $\lambda$ to $\operatorname{trace}^{2}\left(\gamma^{\lambda}\right)$ is holomorphic and nonconstant (see, for example, Bers [B] or $\mathrm{Kra}[\mathrm{Kr} 1])$. But $\operatorname{trace}^{2}\left(\gamma^{\lambda}\right)$ must take on only real values. We have established the required contradiction and completed the proof of Proposition 4.1.

4.4. Let us start with a (not necessarily normalized) sequence of spheres $\bar{S}_{m}, m=1,2,3, \ldots$, of fixed signature $\left(0, n+3 ; \nu_{1}, \ldots, \nu_{n+3}\right), n \geq 1$, satisfying (4.1.2). Thus

$$
\left(S_{m}\right)_{0}=\widehat{\mathbf{C}}-\left\{\lambda_{1}^{(m)}, \ldots, \lambda_{n+3}^{(m)}\right\} .
$$

Assume that $\bar{S}_{n} \rightarrow \bar{S}_{\infty}$, in the sense that

$$
\lim _{m \rightarrow \infty} \lambda_{j}^{(m)}=\lambda_{j}^{(\infty)}
$$

exists for $j=1, \ldots, n+3$, and that $\bar{S}_{\infty}$ is of type $(O, N)$ with $N<n+3$ (thus $\lambda_{j}^{(\infty)}=\lambda_{k}^{(\infty)}$ for some pair of indices $j, k$ with $\left.1 \leq j<k \leq n+3\right)$. We proceed to define the signature of $\bar{S}_{\infty}$. Let $\left\{\lambda_{1}^{(\infty)}, \ldots, \lambda_{N}^{(\infty)}\right\}$ be the maximal subset of $\left\{\lambda_{1}^{(\infty)}, \ldots, \lambda_{n+3}^{(\infty)}\right\}$ consisting of distinct entries, after relabeling the indices if necessary. We say that $\lambda_{j}^{(\infty)}, 1 \leq j \leq N$, is a simple point if $\lambda_{j}^{(\infty)} \neq \lambda_{k}^{(\infty)}$ for $k=N+1, \ldots, n+3$ (thus a single sequence $\left\{\lambda_{j}^{(m)}\right\}$ converges to $\lambda_{j}^{(\infty)}$ ); otherwise $\lambda_{j}^{(\infty)}$ is called a multiple point. Our hypothesis that $N<n+3$ guarantees that there exists at least one multiple point. We set $\mu_{j}=\nu_{j}$ if $\lambda_{j}^{(\infty)}$ is a simple point and $\mu_{j}=\infty$ if $\lambda_{j}^{(\infty)}$ is a multiple point. The signature of $\bar{S}_{\infty}$ is defined as $\left(0, N ; \mu_{1}, \ldots, \mu_{N}\right)$.

Theorem. Let $\varphi_{m}$ be the Fuchsian uniformizing connection for $\bar{S}_{m}, m=1,2$, $\ldots, \infty$. Then

$$
\varphi_{\infty}=\lim _{m \rightarrow \infty} \varphi_{m}
$$


uniformly on compact subsets of $\widehat{\mathbf{C}}$ if $\bar{S}_{\infty}$ has hyperbolic signature, that is, if

$$
\sum_{j=1}^{N}\left(1-\mu_{j}^{-1}\right)>2 \text {. }
$$

Remarks. (1) Recall that for meromorphic functions uniform convergence on compact subsets means uniform convergence of the functions on compact subsets that avoid the poles of all but finitely many functions in the given sequence.

(2) The theorem holds, of course, also when the sequence $\left\{\bar{S}_{m}\right\}$ is not a degenerating sequence (that is, if $\bar{S}_{\infty}$ is of type $(0, n+3)$ ), as a consequence of Proposition 4.1.

4.5. Let $\bar{S}$ be the sphere with hyperbolic signature $\left(0, n+3 ; \nu_{1}, \ldots, \nu_{n+3}\right)$. Then $\bar{S}$ carries a complete metric of constant negative curvature -1 ,

$$
\lambda_{\bar{S}}(z)|d z|^{1}
$$

This metric is defined by projecting the Poincaré metric

$$
\frac{2}{1-|z|^{2}}|d z|
$$

on the unit disk $\Delta$ to $S$ using a branched holomorphic universal covering map $\pi: \Delta \rightarrow S$. Thus we have the formula

$$
\lambda_{\bar{S}}(\pi z)\left|\pi^{\prime}(z)\right|=\frac{2}{1-|z|^{2}}, \quad z \in \Delta .
$$

The metric has singularities at the points of $\bar{S}-S_{0}$. If $z_{0} \in \bar{S}-S_{0}$ is a point of ramification number $\nu<\infty$, then for an appropriate local coordinate $Z$ vanishing at $z_{0}$, we can write the metric as

$$
\frac{2}{\nu} \frac{|Z|^{1 / \nu-1}}{1-|Z|^{2 / \nu}}|d Z|
$$

Similarly, if $\nu=\infty$, then the expression for the metric is

$$
\frac{1}{|Z| \log \left|Z^{-1}\right|}|d Z| \text {. }
$$

(For details, see [Kr2, pp. 72-78].)

It can be shown that

$$
\text { Area } S=\iint_{S} \lambda_{\bar{S}}(z)^{2}\left|\frac{d z \wedge d \bar{z}}{2}\right|=2 \pi\left(-2+\sum_{j=1}^{n+3}\left(1-\nu_{j}^{-1}\right)\right) \geq \frac{\pi}{21} \text {. }
$$

We shall consider the sphere $\bar{S}_{1}$ with signature to be subordinate to the sphere $\bar{S}_{2}$ with signature provided that for every $z \in \widehat{\mathbf{C}}$, its ramification number as a point of $\bar{S}_{1}$ is greater than its ramification number as a point of $\bar{S}_{2}$ (we shall write $\bar{S}_{1} \leq \bar{S}_{2}$ ). We regard $\infty$ to be a multiple of every natural number and unramified points as points with ramification number 1 .

${ }^{1}$ The symbol $\lambda$ is used both for the Poincaré metric and for points in $\widehat{\mathbf{C}}$. The context should make it clear which meaning is intended. 
Lemma. If $\bar{S}_{1} \leq \bar{S}_{2}$, then $\lambda_{\bar{S}_{1}} \geq \lambda_{\bar{S}_{2}}$.

Proof. Let $u_{j}=2 \log \lambda_{\bar{S}_{j}}, j=1,2$. The fact that our metrics have curvature -1 means that

$$
\left(u_{j}\right)_{z \bar{z}}=\frac{1}{2} e^{u_{j}}
$$

The function $u=u_{1}-u_{2}$ is certainly smooth on $\left(S_{1}\right)_{0}$, and satisfies the partial differential equation

$$
u_{z \bar{z}}=\left(u_{1}\right)_{z \bar{z}}-\left(u_{2}\right)_{z \bar{z}}=\frac{1}{2} e^{u_{1}}-\frac{1}{2} e^{u_{2}}=\frac{1}{2} e^{\sigma} u,
$$

where $\sigma$ is between $u_{1}$ and $u_{2}$. It follows that $u$ cannot have a positive maximum or a negative minimum on $\left(S_{1}\right)_{0}$. Now if $x \notin\left(S_{1}\right)_{0}$, let $\nu(\geq 2)$ be the ramification of $\bar{S}_{1}$ at $x$ and $\mu(\leq \nu)$ be the ramification of $\bar{S}_{2}$ at $x$. If $\mu=\nu$, then by (4.5.1) and (4.5.2) $u$ can be extended to be smooth at $x$ and the previous assertion about maxima and minima applies to $x$ as well (by standard results from potential theory). If $\mu<\nu$, then (again by (4.5.1) and $(4.5 .2))$

$$
\lim _{z \rightarrow x} u(z)=+\infty .
$$

Without loss of generality, we may assume that this latter situation occurs at at least one point (otherwise $\bar{S}_{1}=\bar{S}_{2}$ and there is nothing to prove). We conclude that $u$ has a nonnegative minimum.

Remarks. (1) The lemma, in particular, applies to the situation where $\bar{S}_{1}$ is replaced by a domain $D \subset\left(S_{2}\right)_{0}$.

(2) The above lemma is well known. See, for example, Wong [W]. The simple proof was reproduced for the reader's convenience.

4.6. We begin the proof of Theorem 4.4 by considering normalized surfaces; that is, we assume that for $m=1,2,3, \ldots$ (therefore also for $m=\infty$ ), $\lambda_{n+1}^{(m)}=0, \lambda_{n+2}^{(m)}=1$, and $\lambda_{n+3}^{(m)}=\infty$, and furthermore that

$$
\sum_{j=n+1}^{n+3}\left(1-\nu_{j}^{-1}\right)>2 .
$$

Let $\Delta$ be the unit disk. Choose a point $b \in\left(S_{\infty}\right)_{0} \cap \bigcap_{m=1}^{\infty}\left(S_{m}\right)_{0}$ (thus $b$ is not a distinguished point on any of the surfaces in question). For $m=$ $1,2, \ldots, \infty$, let $\pi_{m}: \Delta \rightarrow S_{m}$ be the branched holomorphic universal covering with $\pi_{m}(0)=b$ and $\pi_{m}^{\prime}(0)>0$. Note that for each finite $m$, all solutions of the equation

$$
\pi_{m}(z)=\lambda_{n+j}
$$

have multiplicity $\nu_{n+j}$ (multiplicity $\infty$ means no solutions) for $j=1,2,3, \ldots$. Hence by Schottky's theorem [Hi, p. 250; T, pp. 262-264], the functions $\left\{\pi_{m}\right\}$ form a normal family and by passing to a subsequence we may assume that

$$
\lim _{m \rightarrow \infty} \pi_{m}=\pi
$$


uniformly on compact subsets of $\Delta$. It follows that

$$
\pi(0)=\lim _{m \rightarrow \infty} \pi_{m}(0)=b, \quad \pi^{\prime}(b)=\lim _{m \rightarrow \infty} \pi_{m}^{\prime}(0) \geq 0 .
$$

We must show that $\pi=\pi_{\infty}$. Let $D$ be a domain with

$$
b \in D \subset\left(S_{\infty}\right)_{0} \cap \bigcap_{m=1}^{\infty}\left(S_{m}\right)_{0} .
$$

Let $\tilde{\pi}: \Delta \rightarrow D$ be a holomorphic universal covering map with $\tilde{\pi}(0)=b$. Then by Lemma 4.5 ,

$$
\pi_{m}^{\prime}(0)=2 \lambda_{\bar{S}_{m}}(b)^{-1} \geq 2 \lambda_{D}(b)^{-1}=\left|\tilde{\pi}^{\prime}(0)\right|=\alpha>0 .
$$

It follows that

$$
\pi^{\prime}(0) \geq \alpha,
$$

and hence $\pi$ is a local homeomorphism in a neighborhood of zero. Similarly, with $\bar{S}$ equal to the sphere with $0,1, \infty$ as distinguished points with ramification numbers $\nu_{n+1}, \nu_{n+2}, \nu_{n+3}$, and $\tilde{\pi}: \Delta \rightarrow S$ the corresponding branched holomorphic universal covering with $\tilde{\pi}(0)=b$, we have

$$
\pi_{m}^{\prime}(0)=2 \lambda_{\bar{S}_{m}}(b)^{-1} \leq 2 \lambda_{\bar{S}}(b)^{-1}=\left|\tilde{\pi}^{\prime}(0)\right|=\beta<\infty .
$$

It follows that

$$
\pi^{\prime}(0) \leq \beta
$$

Let $D$ be any simply connected domain with $b \in D$ and $\operatorname{cl}(D) \subset\left(S_{\infty}\right)_{0}$. Then $\operatorname{cl}(D) \subset\left(S_{m}\right)_{0}$ for all $m$ sufficiently large. Let $f_{m}$ be an inverse to $\pi_{m}$ defined on $D$ with $f_{m}(b)=0$. Then $\left\{f_{m}\right\}$ forms a normal family on $D$ and (passing to a subsequence) we may assume that

$$
f(z)=\lim _{m \rightarrow \infty} f_{m}(z), \quad z \in D
$$

with $f$ holomorphic on $D$. Since $f_{m}(b)=0$ for all $m$, we conclude that $f(b)=0$. Since

$$
\left|f_{m}^{\prime}(b)\right|=\left|\pi_{m}^{\prime}(0)\right|^{-1} \geq 1 / \beta>0,
$$

we conclude that $f$ is not constant $\left(\left|f^{\prime}(b)\right| \geq 1 / \beta\right)$ and hence

$$
|f(z)|<1, \quad \text { all } z \in D \text {. }
$$

Thus $\pi \circ f$ is always defined and we conclude from $\pi_{m} \circ f_{m}=I$ on $D$ that $f$ is an inverse to $\pi$ on $D$. Since an arbitrary point of $\left(S_{\infty}\right)_{0}$ can be included in $D$, we conclude that $\pi$ covers $\left(S_{\infty}\right)_{0}$.

Our next claim is that $\pi$ is unramified over $\left(S_{\infty}\right)_{0}$. Let $a \in\left(S_{\infty}\right)_{0}$ and choose a neighborhood $D_{1}$ of $a$ in $\left(S_{\infty}\right)_{0}$. We may assume that $D_{1}$ is in $\left(S_{m}\right)_{0}$ for all sufficiently large $m$. Choose $z_{0} \in \Delta$ with $\pi\left(z_{0}\right)=a$. Let $D_{0}$ be a relatively compact neighborhood of $a$ in $D_{1}$. Then (as above)

$$
\begin{aligned}
\left|\pi^{\prime}\left(z_{0}\right)\right| & =\lim _{m \rightarrow \infty}\left|\pi_{m}^{\prime}\left(z_{0}\right)\right|=\lim _{m \rightarrow \infty} \frac{2}{1-\left|z_{0}\right|^{2}} \lambda_{\bar{S}_{m}}\left(\pi_{m} z_{0}\right)^{-1} \\
& \geq \frac{2}{1-\left|z_{0}\right|^{2}} \lim _{m \rightarrow \infty} \lambda_{D_{1}}\left(\pi_{m} z_{0}\right)^{-1} \geq \frac{2}{1-\left|z_{0}\right|^{2}} \inf _{\zeta \in D_{0}}\left\{\lambda_{D_{1}}(\zeta)^{-1}\right\}>0 .
\end{aligned}
$$


We next show that every curve in $\left(S_{\infty}\right)_{0}$ can be lifted to $\Delta$ (via $\pi$ ). Let $\alpha:[0,1] \rightarrow\left(S_{\infty}\right)_{0}$ be a path with $\alpha(0) \stackrel{\infty}{=} b$ (without loss of generality). We choose a partition $0=t_{0}<t_{1}<t_{2}<\cdots<t_{k}=1$ of $[0,1]$ so that $\alpha\left(\left[t_{i}, t_{i+1}\right]\right)$ is contained in a disk $D_{i}$ contained in all but finitely many $\left(S_{m}\right)_{0}$ for $i=$ $0, \ldots, k-1$. It follows that $D_{i} \cap D_{i+1}$ is not empty for $i=0, \ldots, k-2$. In $D=D_{0}$ we know that $f_{m}$ converges uniformly to $f$ and hence we can lift $\alpha \mid\left[0, t_{1}\right]$ to $\Delta$ by $f$ to a path beginning at 0 . Let $b_{1}=\alpha\left(t_{1}\right)$. Then $b_{1} \in D_{0} \cap D_{1}$ and $\lim _{m \rightarrow \infty} f_{m}\left(b_{1}\right)=f\left(b_{1}\right)$. It follows that we can construct local inverses $f_{m}^{(1)}$ to $\pi_{m}$ in $D_{1}$ with the property that $\lim _{m \rightarrow \infty} f_{m}^{(1)}=f^{(1)}$ uniformly on compact subsets of $D_{1}$. It folows that $\alpha \mid\left[0, t_{2}\right]$ lifts to $\Delta$. By induction we can finish this process. Let $z_{0}$ now be any point lying over $b$ in $\Delta$ (via $\pi$ ). We want to lift $\alpha$ to a curve in $\Delta$ beginning at $z_{0}$. Choose any path $\tilde{d}$ in $\pi^{-1}\left(\left(S_{m}\right)_{0}\right)$ joining $z_{0}$ to 0 and let $d=\pi(\tilde{d})$. Let $\tilde{\alpha}$ be a lift of $-d+\alpha$ beginning at 0 . Then $\tilde{d}+\tilde{\alpha}$ is a lift of $\alpha$ beginning at $z_{0}$.

It remains to verify that $\pi$ is a branched holomorphic universal covering map of $S_{\infty}$ corresponding to the limiting signature. For then the limit $\lim _{m \rightarrow \infty} \varphi_{m}=\lim _{m \rightarrow \infty}\left\{f_{m}, \cdot\right\}=\{f, \cdot\}=\varphi$ is independent of any subsequences chosen, and so the entire sequence converges.

4.7. We proceed to study the monodromy maps

$$
\begin{aligned}
& \eta_{m}^{*}: \pi_{1}\left(\left(S_{m}\right)_{0}, b\right) \rightarrow \operatorname{PSL}(2, \mathbf{C}), \quad m=1,2,3, \ldots, \\
& \eta^{*}: \pi_{1}\left(\left(S_{\infty}\right)_{0}, b\right) \rightarrow \operatorname{PSL}(2, \mathbf{C}),
\end{aligned}
$$

induced by the connections $\varphi_{m}$ and $\varphi$. To specify the map $\eta_{m}^{*}$ we must specific the initial function that solves

$$
\left\{f_{m}, \cdot\right\}=\varphi_{m}
$$

in a neighborhood of $b$. We choose for $f_{m}$ the inverse of $\pi_{m}$ with $f_{m}(b)=0$. Similarly, for $\eta$. It follows that

$$
\begin{aligned}
\Gamma_{m} & =\operatorname{im} \eta_{m}^{*}=\left\{\gamma \in \operatorname{PSL}(2, \mathbf{R}) ; \pi_{m} \circ \gamma=\pi_{m}\right\}, \quad m=1,2,3, \ldots, \\
\Gamma & =\operatorname{im} \eta^{*}=\{\gamma \in \operatorname{PSL}(2, \mathbf{R}) ; \pi \circ \gamma=\pi\} .
\end{aligned}
$$

Note that by our previous arguments $\left\{f_{m}\right\}$ converges to $f$ (by passing to a subsequence, if necessary).

We show next that

$$
\pi: \pi^{-1}\left(\left(S_{\infty}\right)_{0}\right) \rightarrow\left(S_{\infty}\right)_{0}
$$

is a Galois covering with $\Gamma$ as the group of deck transformations. Let $z_{0}$ and $z_{1}$ be two points in $(\pi)^{-1}\left(\left(S_{\infty}\right)_{0}\right)$ and assume $\pi\left(z_{0}\right)=\pi\left(z_{1}\right)$. Join $z_{0}$ to $z_{1}$ by a path $\tilde{\alpha}$ in $(\pi)^{-1}\left(\left(S_{\infty}\right)_{0}\right)$. Let $\alpha=\pi(\tilde{\alpha})$. Join $b$ to $\pi\left(z_{0}\right)$ by a path $d$ in $\left(S_{\infty}\right)_{0}$. Continuing $f$ around $d+\alpha-d$ leads to the function $\gamma \circ f$ with $\gamma \in \Gamma$. Since we may take $f$ to be defined also at $\pi\left(z_{0}\right)$, we see that $f$ continued around $\alpha$ leads to $\gamma \circ f$. Thus $\gamma\left(z_{0}\right)=z_{1}$. The converse is trivial. 
It remains to determine the signature of $\Gamma$. Let $\alpha_{j}, j=1, \ldots, n+3$, be a simple closed curve in $\left(S_{m}\right)_{0}$ that begins at $b$ and has winding number +1 around $\lambda_{j}$, and winding number 0 around $\lambda_{i}, i \neq j$. Then $\eta_{m}^{*}\left(\alpha_{j}\right)$ is elliptic of order $\nu_{j}$ if $\nu_{j}<\infty$ and parabolic of $\nu_{j}=\infty$. Furthermore,

$$
\Gamma_{m}=\left\langle\eta_{m}^{*}\left(\alpha_{1}\right), \ldots, \eta_{m}^{*}\left(\alpha_{n+3}\right)\right\rangle
$$

Remark. We could choose $\alpha_{1}, \ldots, \alpha_{n+3}$ to be a canonical basis for $\pi_{1}\left(\left(S_{m}\right)_{0}, b\right)$ in the sense that

$$
\alpha_{1} \cdot \alpha_{2} \cdots \cdot \alpha_{n+3}=1 \text {. }
$$

Here - is the group operation in the fundamental group. Then $\Gamma_{m}$ has the presentation

$$
\eta_{m}^{*}\left(\alpha_{j}\right)^{\nu_{j}}=I, \quad j=1, \ldots, n+3,
$$

(as usual, if $\nu_{j}=\infty$, the above equation is to be interpreted that $\eta_{m}^{*}\left(\alpha_{j}\right)$ is parabolic) and

$$
\eta_{m}^{*}\left(\alpha_{n+3}\right) \circ \cdots \circ \eta_{m}^{*}\left(\alpha_{2}\right) \circ \eta_{m}^{*}\left(\alpha_{1}\right)=I \text {. }
$$

Consider next a simple point $\lambda_{j}^{(\infty)} \in \widehat{\mathbf{C}}-\left(S_{\infty}\right)_{0}$. In this case (note that $1 \leq j \leq N)$

$$
\operatorname{trace}^{2} \eta^{*}\left(\alpha_{j}\right)=\lim _{m \rightarrow \infty} \operatorname{trace}^{2} \eta_{m}^{*}\left(\alpha_{j}\right)
$$

Since the terms in the sequence are constant, $\lambda_{j}$ is a point of ramification order $\nu_{j}$

Now assume that $\lambda_{j}^{(\infty)} \in \widehat{\mathbf{C}}-\left(S_{\infty}\right)_{0}$ is a multiple point. Thus for some $k$, $N<k \leq n+3$,

$$
\lambda_{j}^{(\infty)}=\lim _{m \rightarrow \infty} \lambda_{j}^{(m)}=\lim _{m \rightarrow \infty} \lambda_{k}^{(m)} .
$$

We choose a closed curve $\alpha$ beginning at $b$ with the following properties. For sufficiently large $m, \alpha \subset\left(S_{\infty}\right)_{0} \cap\left(S_{m}\right)_{0}, \alpha$ has winding number +1 around $\lambda_{j}^{(\infty)}, \lambda_{j}^{(m)}, \lambda_{k}^{(\infty)}$, and all other $\lambda_{i}^{(m)}$ that converge to $\lambda_{j}^{(\infty)}$. Then $\eta_{m}^{*}(\alpha)$ is hyperbolic for $m=1,2,3, \ldots$, and

$$
\lim _{m \rightarrow \infty} \eta_{m}^{*}(\alpha)=\eta^{*}(\alpha)
$$

We must show that $\eta^{*}(\alpha)$ is parabolic. For each $m$, let $\beta_{m}$ be the family of curves in $\left(S_{m}\right)_{0}$ freely homotopic to $\alpha$. The limit of the extremal lengths of these families is zero. It follows that

$$
\lim _{m \rightarrow \infty} \operatorname{trace}^{2} \eta_{m}^{*}(\alpha)=4
$$

(see Maskit [Mt, Lemma 1]). We must eliminate the possibility that $\eta^{*}(\alpha)$ is the identity. If $\eta^{*}(\alpha)$ were the identity, then $\lambda=\lambda_{j}^{(\infty)}$ would be assumed by $\pi$ somewhere, say at $z_{0} \in \Delta$. It would follow that $\pi^{\prime}\left(z_{0}\right) \neq 0$ (otherwise $\eta^{*}(\alpha)$ would have to be elliptic). Hence we could choose a circle $\alpha$ around $z_{0}$ 
(positively oriented) so that $\pi(z)=\lambda$ has a unique solution in the closed disk bounded by $\alpha$. It follows that

$$
1=\frac{1}{2 \pi i} \int_{\alpha} \frac{\pi^{\prime}(z) d z}{\pi(z)-\lambda} .
$$

Since $\pi_{m} \rightarrow \pi$ uniformly on a neighborhood of $\alpha$, we can choose $m_{0}$ so that $m \geq m_{0}$ implies that $\pi_{m}(z) \neq \lambda_{j}^{(\infty)}$ for $z \in \alpha$. Let $\epsilon=\min \{|\pi(z)-\lambda| ; z \in \alpha\}$. Then $\varepsilon>0$. Since $\pi_{m} \rightarrow \pi$ uniformly on $\alpha$, we can adjust $m_{0}$ such that $m \geq m_{0}$ implies

$$
\left|\pi_{m}(z)-\pi(z)\right| \leq \varepsilon / 2 \text { on } \alpha .
$$

It follows that

$$
\left|\pi_{m}(z)-\lambda\right| \geq \varepsilon / 2 \text { on } \alpha \text { for } m \geq m_{0}
$$

and hence there exists an $m_{1}$ with

$$
1=\frac{1}{2 \pi i} \int_{\alpha} \frac{\pi_{m}^{\prime}(z) d z}{\pi_{m}(z)-\lambda} \quad \text { for } m \geq m_{1} .
$$

It follows that for $m \geq m_{1}, \pi_{m}$ takes on the value $\lambda$ precisely once inside $\alpha$, and hence also every value in $|z-\lambda| \leq \varepsilon / 2$. But $\lim _{m \rightarrow \infty} \lambda_{j}^{(m)}=\lambda$ and $\lambda_{j}^{(m)}$ is a point of multiplicity $\nu_{j}$. We have arrived at a contradiction. It follows that $\pi=\pi_{\infty}, f=f_{\infty}$, and $\eta^{*}=\eta_{\infty}^{*}$. This completes the proof of Theorem 4.4 when the surfaces are normalized and the signature satisfies (4.6.1). We shall prove the general case in $\S 4.9$.

Remark. We can use Chuckrow's theorem [C] to conclude that $\eta^{*}(\alpha)$ must be parabolic; see §4.9. The use of Chuchrow's theorem [C] will allow us to give an alternate and shorter proof of parts of Theorem 4.4 in the general setting. We have included the above arguments because they are elementary and depend only on classical theorems. Here we use only Schottky's theorem and extremal lengths. For the general case, we still need extremal lengths. We also will need to know about presentations of Fuchsian groups and convergence properties for sequences of Fuchsian groups.

4.8. We have proved more than we claimed in Theorem 4.4 (for normalized surfaces with (4.6.1)). Let

$$
b \in\left(S_{\infty}\right)_{0} \cap\left(S_{m}\right)_{0}, \quad m=1,2,3, \ldots
$$

Choose the branched holomorphic universal covering map

$$
\pi_{m}: \Delta \rightarrow S_{m}, \quad m=1,2,3, \ldots, \infty,
$$

with

$$
\pi_{m}(0)=b, \quad \pi_{m}^{\prime}(0)>0 .
$$

Then $\pi_{m}$ approaches $\pi_{\infty}$ uniformly on compact subsets of $\Delta$.

Remark. For (unbranched) holomorphic universal coverings, this result has been obtained previously by Hejhal [Hel].

We have also established the following proposition. 
Proposition. Let $\lambda_{m}(z)|d z|$ be the Poincaré metric for $\bar{S}_{m}, m=1,2,3, \ldots$, $\infty$. Then

$$
\lambda_{\infty}(z)=\lim _{m \rightarrow \infty} \lambda_{m}(z), \quad \text { all } z \in\left(S_{\infty}\right)_{0},
$$

the limit is uniform on compact subsets.

Proof. Let $D$ be a simply connected domain in $\left(S_{\infty}\right)_{0}$, with $b \in D$ (as in the proof of Theorem 4.4). Let $f_{m}$ be the inverse of $\pi_{m}$ with $f_{m}(b)=0$. Then for any $z \in D$,

$$
\lambda_{m}(z)=\frac{2}{1-\left|f_{m}(z)\right|^{2}}\left|f_{m}^{\prime}(z)\right| .
$$

The proposition now follows from the convergence of $\left\{f_{m}\right\}$ to $f_{\infty}$.

4.9. We return to the general situation treated in $\S 4.4$. We choose $b \in$ $\left(S_{\infty}\right)_{0} \cap \cap_{m=1}^{\infty}\left(S_{m}\right)_{0}$ and the unique normalized branched holomorphic coverings $\pi_{m}(m=1,2,3, \ldots, \infty)$ discussed in $\S 4.6$. We cannot claim that $\left\{\pi_{m}\right\}$ is a normal family. However, the normalized sequence of inverses $\left\{f_{m}\right\}$ discussed in $\S 4.6$ is indeed a normal family. Since $f_{m}$ is univalent on $D$, by Hurwitz's theorem, we may assume (after passing to a subsequence) that $\lim _{m \rightarrow \infty} f_{m}=f$ is either univalent or constant.

Lemma. The limiting function $f$ is constant if and only if the limiting surface $\bar{S}_{\infty}$ is elementary.

Before proving the lemma, we must discuss convergence of the Fuchsian groups we are investigating.

Let $\Gamma_{m}$ be the covering group of $\pi_{m}, m=1,2,3, \ldots, \infty$. The group $\Gamma_{m}$ is canonically isomorphic to $\Gamma_{1}$ for $m=1,2, \ldots$, while $\Gamma_{\infty}$ is canonically isomorphic to a subgroup of $\Gamma_{1}$ (necessarily of the second kind). To be specific, we let $\chi_{m}: \Gamma_{1} \rightarrow \Gamma_{m}$ be the isomorphism that sends $\eta_{1}^{*}\left(\alpha_{j}\right)$ to $\eta_{m}^{*}\left(\alpha_{j}\right), j=$ $1, \ldots, n+3$. Note that the $\alpha_{j}$ 's are different curves on the different surfaces $\left(S_{m}\right)_{0}$; but this causes no difficulty. For each $\lambda \in \widehat{\mathbf{C}}-\left(S_{\infty}\right)_{0}$, we introduce a curve $\tilde{\alpha}$ as follows. If $\lambda$ is a simple point, we use a curve $\alpha_{j}$ as above. If $\lambda$ is a multiple point, then we choose a simple curve $\alpha$ that has winding number +1 around $\lambda$ and around the points $\lambda_{j}^{(m)}$ that converge to $\lambda$. In this way we obtain $N$ curves $\tilde{\alpha}_{1}, \ldots, \tilde{\alpha}_{N}$. The group $\Gamma_{m}^{0}$ is then defined as

$$
\Gamma_{m}^{0}=\left\langle\eta_{m}^{*}\left(\tilde{\alpha}_{1}\right), \ldots, \eta_{m}^{*}\left(\tilde{\alpha}_{N}\right)\right\rangle \text {. }
$$

Note that $\eta_{m}^{*}\left(\tilde{\alpha}_{j}\right)$ is parabolic or elliptic if $\tilde{\alpha}_{j}$ corresponds to a simple point and that $\eta_{m}^{*}\left(\tilde{\alpha}_{j}\right)$ is hyperbolic if $\tilde{\alpha}_{j}$ corresponds to a multiple point. There exists an obvious isomorphism $\chi_{\infty}$ of $\Gamma_{1}^{0}$ onto $\Gamma_{\infty}$. Note that $\chi_{m}\left(\Gamma_{1}^{0}\right)=\Gamma_{m}^{0}$.

Let $\gamma \in \Gamma_{1}$ be arbitrary. Consider the sequence of Möbius transformations $\chi_{m}(\gamma), m=1,2,3, \ldots$. Since these form a normal family, we have

$$
\lim _{m \rightarrow \infty} \chi_{m}(\gamma)=\chi(\gamma), \quad \text { all } \gamma \in \Gamma_{1}^{0},
$$

where $\chi$ is the homomorphism induced by $f$. 
We shall see that $\chi(\gamma)=\chi_{\infty}(\gamma)$ for all $\gamma \in \Gamma_{1}^{0}$ and that $\chi_{m}(\gamma)$ converges to a constant (of absolute value 1) if $\gamma \in \Gamma_{1}-\Gamma_{1}^{0}$. (We already have this result for the normalized case for which Theorem 4.4 has been proved.)

Proof of Lemma (if part). Let $\alpha$ be a closed path starting at $b$ and contained in $\left(S_{\infty}\right)_{0} \cap \bigcap_{m=1}^{\infty}\left(S_{m}\right)_{0}$. Since each $f_{m}$ can be continued analytically along $\alpha$, so can $f$. We consider the group $\Gamma_{1}^{0}$ defined above and the isomorphisms $\chi_{m}: \Gamma_{1}^{0} \rightarrow \operatorname{PSL}(2, \mathbf{R}), m=1,2,3, \ldots, \infty$. Now $\Gamma_{1}^{0}$ is elementary if and only if $\bar{S}_{\infty}$ is. If $f$ were constant, then $\chi$ of (4.9.1) would be the trivial homomorphism. By Chuckrow's theorem [C], $\Gamma_{1}^{0}$ (and hence also $\bar{S}_{\infty}$ ) would have to be elementary.

Proof of Theorem 4.4 (conclusion). We start by using the information that $\Gamma_{1}^{0}$ is nonelementary. Hence $f$ is univalent. By Jørgensen's extension [J] of Chuckrow's theorem [C], $\chi$ is an isomorphism of $\Gamma_{1}^{0}$ onto the finitely generated Fuchsian group $\chi\left(\Gamma_{1}^{0}\right)$.

We now construct an isomorphism $\theta$ of $\Gamma_{\infty}$ onto $\chi\left(\Gamma_{1}^{0}\right)$ that is induced by the map $f \circ \pi_{\infty}$. Let $\gamma \in \Gamma_{\infty}$. Choose a path $\alpha$ in $\Delta$ from 0 to $\gamma(0)$ that avoids elliptic fixed points. Let $\tilde{\alpha}=\pi_{\infty}(\alpha)$, and let $\theta(\gamma)$ be the element of $\operatorname{PSL}(2, \mathbf{R})$ obtained by continuing $f$ along $\tilde{\alpha}$. Let $\tilde{f}$ be the germ of $f \circ \pi_{\infty}$ at the origin. Let $\Delta_{0}$ be the complement in $\Delta$ of the elliptic fixed points of $\Gamma_{\infty}$. It is obvious that $\tilde{f}$ can be continued analytically over all of $\Delta_{0}$. We want to show that $\tilde{f}$ can be continued as a single-valued locally univalent function: first to $\Delta_{0}$, and finally to all of $\Delta$. Let $z_{0} \in \Delta$ be an elliptic fixed point of order $\nu$ of $\Gamma_{\infty}$. Let $\alpha$ be a small circle around $z_{0}$. Then $\pi_{\infty}(\alpha)$ is the $\nu$ th power of a small circle around $\pi_{\infty}\left(z_{0}\right)$. Continuing $f$ around such a curve leads back to $f$. We have produced the extension to $\Delta_{0}$ and indeed to all of $\Delta$. The extension is clearly holomorphic. We claim next that

$$
\tilde{f} \circ \gamma=\theta(\gamma) \circ \tilde{f}, \quad \text { all } \gamma \in \Gamma_{\infty} \text {. }
$$

It suffices to verify (4.9.2) in a neighborhood of 0 . In such a neighborhood, $\tilde{f}=f \circ \pi_{\infty}$ and $\theta(\gamma) \circ \tilde{f}$ is $\tilde{\tilde{f}} \circ \pi_{\infty}$, where $\tilde{\tilde{f}}$ is the result of continuing $f$ along $\tilde{\alpha}$. On the other hand, $\tilde{f} \circ \gamma$ is the result of continuing $f \circ \pi_{\infty} \circ \gamma=f \circ \pi_{\infty}$ along $\tilde{\alpha}$. These two functions are clearly the same. The function $\tilde{f}$ is locally univalent on $\Delta_{0}$. We must show that $\tilde{f}^{\prime}\left(z_{0}\right) \neq 0$ for every elliptic fixed point $z_{0} \in \Delta$ of $\Gamma_{\infty}$. Let (without loss of generality) $\pi_{\infty}\left(z_{0}\right)=\lambda_{1}^{(\infty)}$. Then $\lambda_{1}^{(\infty)}$ is a simple point and

$$
\lambda_{1}^{(\infty)}=\lim _{m \rightarrow \infty} \lambda_{1}^{(m)}
$$

where $\lambda_{1}^{(m)}$ is the image under $\pi_{m}$ of an elliptic fixed point of $\Gamma_{m}$. Then (as 
in $\S 4.7)$, using the closed curve $\alpha$ introduced above

$$
\begin{aligned}
\operatorname{ord}_{z_{0}}\left(\tilde{f}-\lambda_{1}^{(\infty)}\right) & =\frac{1}{2 \pi i} \int_{\alpha} \frac{\tilde{f}^{\prime}(z)}{\tilde{f}(z)-\lambda_{1}^{(\infty)}} d z \\
& =\frac{1}{2 \pi} \int_{\alpha} d\left(\arg \left(f \circ \pi_{\infty}-\lambda_{1}^{(\infty)}\right)\right) \\
& =\frac{1}{2 \pi} \lim _{m \rightarrow \infty} \int_{\alpha} d\left(\arg \left(f_{m} \circ \pi_{\infty}-\lambda_{1}^{(m)}\right)\right) \\
& =\frac{1}{\nu} \nu=1,
\end{aligned}
$$

where $\nu$ is the order of the stabilizer of the preimages of $\lambda_{1}^{(m)}$ for $m=$ $1,2,3, \ldots, \infty$. A similar modification of the arguments of $\S 4.7$ shows that $\theta$ takes parabolic elements of $\Gamma_{\infty}$ to parabolic elements of $\chi\left(\Gamma_{1}^{0}\right)$. Alternatively, by these methods we can estimate the growth of $\tilde{f}$ in the cusped regions determined by the punctures on $\Delta / \Gamma_{\infty}$. We finally compute

$$
\{\tilde{f}, z\}=\left\{f, \pi_{\infty}(z)\right\}\left(\pi_{\infty}\right)^{\prime}(z)^{2}+\left\{\pi_{\infty}, z\right\} .
$$

By $\S 1.5,\{\tilde{f}, \cdot\}$ is a cusp form for $\Gamma_{\infty}$ (its projection to $\bar{S}_{\infty}$ has only simple poles). By the results of [Krl], $\tilde{f}: \Delta \rightarrow \Delta$ is a covering map. Hence a Möbius transformation. The normalizations of $\pi_{\infty}$ and $f$ force $\tilde{f}$ to be the identity. Thus, in particular, $f=f_{\infty}$.

This argument does not yet show that $\lim _{m \rightarrow \infty} \pi_{m}=\pi_{\infty}$. We note that from the convergence of $\left\{f_{m}\right\}$ to $f_{\infty}$, we conclude (using Koebe's $\frac{1}{4}$-theorem) that for some $\varepsilon>0$, the image of $f_{m}$ (for each $m$ ) contains the disk of radius $\varepsilon$. Thus $\pi_{m}$ is a normal family on this disk and converges uniformly to $\pi_{\infty}$. We consider an arbitrary point $z_{0} \in \Delta$ that is not an elliptic fixed point of $\Gamma_{\infty}$. Then $\pi_{\infty}\left(z_{0}\right) \in\left(S_{\infty}\right)_{0}$ and a path $\alpha$ in $\Delta_{0}$ connecting 0 to $z_{0}$ projects to a path in $\tilde{\alpha}$ in $\left(S_{\infty}\right)_{0}$. We continue the functions $f_{m}, m=1,2,3, \ldots, \infty$ along $\tilde{\alpha}$ and obtain (after passing to a subsequence) a sequence of functions $\left\{f_{m}\right\}$ that converges to $f_{\infty}$ (we are using the same symbol for the function and its analytic continuation) in a neighborhood of $\pi_{\infty}\left(z_{0}\right)$. It follows that $\left\{\pi_{m}\right\}$ is a normal family in a neighborhood of $z_{0}$. This shows that $\left\{\pi_{m}\right\}$ is a normal family on $\Delta_{0}$, and hence on all of $\Delta$.

Remark. We have now completed the proof of Theorem 4.4 and its consequences (in the general case) given in $\S 4.8$. It remains to finish proving the only if part of the lemma. This will be donel in $\S 4.10$.

We record the convergence properties we obtained in the following

Proposition. Under the normalization for the covering maps

$$
\pi_{m}: \Delta \rightarrow S_{m}, \quad m=1,2,3, \ldots, \infty,
$$

given by

$$
\pi_{m}(0)=b, \quad \pi_{m}^{\prime}(0)>0
$$


where $b \in\left(S_{\infty}\right)_{0} \cap \bigcap_{m=1}^{\infty}\left(S_{m}\right)_{0}$, the sequence $\left\{\pi_{m}\right\}$ converges uniformly on compact subsets of $\Delta$ to $\pi_{\infty}$, and the sequence of groups $\left\{\Gamma_{m}^{0}\right\}$ converges algebraically to $\Gamma_{\infty}$.

4.10. Assume now that $\Gamma_{0}$ (and hence also $\bar{S}_{\infty}$ ) is elementary and that $f$ is not constant. In this case, we can proceed exactly as above with a branched covering map $\pi_{\infty}$ which is now defined either on $\mathbf{C}$ or $\widehat{\mathbf{C}}$ (depending, of course, on the signature of $\bar{S}_{\infty}$ ). Now $\tilde{f}=f \circ \pi_{\infty}$ continues to a nonconstant bounded analytic function on $\mathbf{C}$ or $\widehat{\mathbf{C}}$. We have obtained a contradiction to Liouville's theorem.

4.11. We now construct an example to show that the normalizations used are critical in proving our theorem. Let $\bar{S}_{m}$ be the sphere with ramification points $\lambda_{m}, 0,1, \infty$ and signature $\left(0,4 ; \nu_{1}, \nu_{2}, \nu_{3}, \nu_{4}\right)$. Assume that $0<\lambda_{m}<1$, that $\nu_{2}^{-1}+\nu_{3}^{-1}+\nu_{4}^{-1}<1$, that $2 \leq \nu_{1}<\infty, 3 \leq \nu_{2}<\infty$, and that $\lim _{m \rightarrow \infty} \lambda_{m}=0$. We know that

$$
\lim _{m \rightarrow \infty} \varphi_{m}=\varphi_{\infty},
$$

the Fuchsian uniformizing connection for the thrice punctured sphere. Let us choose $\pi_{m}: \Delta \rightarrow S_{m}$ so that $\pi_{m}(0)=0$. Without loss of generality (rotate $\pi_{m}$ ) we may assume that $\pi_{m}\left(x_{m}\right)=\lambda_{m}$, for some $x_{m}$ with $0<x_{m}<1$ and $x_{m}$ is the nearest point to 0 that gets mapped onto $\lambda_{m}$. The surface $S_{m}$ is fixed under conjugation $j: z \rightarrow \bar{z}$, and hence $j$ lifts to an involution (also $j$ ) that conjugates $\Gamma_{m}$ into itself. It follows that $\left[0, x_{m}\right]$ is mapped injectively by $\pi_{m}$ onto $\left[0, \lambda_{m}\right]$. Further, the closed segment $\left[0, \lambda_{m}\right]$ is the geodesic in $S_{m}$ joining 0 to $\lambda_{m}$. A theorem of Marden [Mn] guarantees the existence of an $x>0$ such that $x_{m} \geq x$ for $m=1,2,3, \ldots$.

It follows that the closed interval $[0, x]$ is mapped by $\pi_{m}$ into $\left[0, \lambda_{m}\right]$. If $\pi$ is a limit of a subsequence of $\left\{\pi_{m}\right\}$, then $\pi$ maps $[0, x]$ into $\bigcap_{m=1}^{\infty}\left[0, \lambda_{m}\right]=$ $\{0\}$. It follows that $\pi \equiv 0$. Nevertheless, the Schwarzians of the inverses of the $\pi_{m}$ converge uniformly to $\varphi_{\infty}$.

4.12. Let $\lambda=\left(\lambda_{1}, \ldots, \lambda_{n}\right) \in W^{n}$ and let us consider $\bar{S}_{\lambda}$, the sphere with distinguished points $\lambda_{1}, \ldots, \lambda_{n}, 0,1, \infty$ and signature

$$
\left(0, n+3 ; \nu_{1}, \nu_{2}, \ldots, \nu_{n}, \nu, \nu, \nu\right) \text {. }
$$

Let $\varphi_{\lambda}$ be Fuchsian uniformizing connection for $\bar{S}_{\lambda}$ and $c(\lambda) \in \mathbf{C}^{n}$ the vector of accessory parameters for this uniformization. Let $A \in \operatorname{PSL}(2, \mathrm{C})$ fix as a set $\{0,1, \infty\}$. (There are only six such elements $A: A_{0}=\left(\begin{array}{ll}1 & 0 \\ 0 & 1\end{array}\right), A_{1}=\left(\begin{array}{ll}0 & -1 \\ 1 & -1\end{array}\right)$, $A_{2}=\left(\begin{array}{ll}-1 & 1 \\ -1 & 0\end{array}\right), A_{3}=\left(\begin{array}{ll}0 & 1 \\ 1 & 0\end{array}\right), A_{4}=\left(\begin{array}{ll}1 & -1 \\ 0 & -1\end{array}\right), A_{5}=\left(\begin{array}{ll}-1 & 0 \\ -1 & 1\end{array}\right)$. This group is isomorphic to the permutation group on three letters, and is generated by the two involutions $\left(\begin{array}{ll}0 & 1 \\ 1 & 0\end{array}\right)$ and $\left(\begin{array}{ll}1 & -1 \\ 0 & -1\end{array}\right)$, for example.) If $\pi$ covers $S_{\lambda}$, then $A^{-1} \circ \pi$ covers $S_{A^{-1}(\lambda)}$. It follows that

$$
\varphi_{A^{-1}(\lambda)}=\left(\varphi_{\lambda} \circ A\right)\left(A^{\prime}\right)^{2} .
$$


Recall that

$$
\begin{aligned}
\varphi_{\lambda}(z)= & \frac{1}{2}\left(1-\nu^{-2}\right) \frac{z^{2}-z+1}{z^{2}(z-1)^{2}} \\
& +\sum_{j=1}^{n} \frac{\lambda_{j}\left(\lambda_{j}-1\right)}{z(z-1)\left(z-\lambda_{j}\right)}\left[\frac{1}{2}\left(1-\nu_{j}^{-2}\right) \frac{1}{z-\lambda_{j}}+c_{j}(\lambda)\right] .
\end{aligned}
$$

Further, we already observed that

$$
\Phi(z)=\frac{1}{2}\left(1-\nu^{-2}\right)\left(\left(z^{2}-z+1\right) / z^{2}(z-1)^{2}\right)
$$

satisfies

$$
(\Phi \circ A)\left(A^{\prime}\right)^{2}=\Phi
$$

Abbreviate $A^{-1}$ by $B$ and conclude from (4.12.1) and from [Kr4, §1] that

$$
\begin{aligned}
A^{\prime}(z) & \sum_{j=1}^{n} \frac{\lambda_{j}\left(\lambda_{j}-1\right)}{(A(z))(A(z)-1)\left(A(z)-\lambda_{j}\right)}\left[\frac{1}{2}\left(1-\nu_{j}^{-2}\right) \frac{1}{A(z)-\lambda_{j}}+c_{j}(\lambda)\right] \\
= & \sum_{j=1}^{n} \frac{\left(B\left(\lambda_{j}\right)\right)\left(B\left(\lambda_{j}\right)-1\right)}{z(z-1)\left(z-B\left(\lambda_{j}\right)\right)}\left[\frac{1}{2}\left(1-\nu_{j}^{-2}\right) \frac{1}{z-B\left(\lambda_{j}\right)}+c_{j}(B(\lambda))\right] \\
= & \sum_{j=1}^{n} \frac{\left(B\left(\lambda_{j}\right)-B \circ A(0)\right)\left(B\left(\lambda_{j}\right)-B \circ A(1)\right)}{(B \circ A(z)-B \circ A(0))(B \circ A(z)-B \circ A(1))\left(B \circ A(z)-B\left(\lambda_{j}\right)\right)} \\
& \times\left[\frac{1}{2}\left(1-\nu_{j}^{-2}\right) \frac{1}{B \circ A(z)-B\left(\lambda_{j}\right)}+c_{j}(B(\lambda))\right] \\
= & \sum_{j=1}^{n} \frac{\left(\lambda_{j}-A(0)\right)\left(\lambda_{j}-A(1)\right)}{(A(z)-A(0))(A(z)-A(1))\left(A(z)-\lambda_{j}\right)} \frac{B^{\prime}\left(\lambda_{j}\right)^{1 / 2}(A(z))^{3 / 2}}{1} \\
& \times\left[\frac{1}{2}\left(1-\nu_{j}^{-2}\right) \frac{1}{A(z)-\lambda_{j}} \frac{\left(B^{\prime}(A z)^{1 / 2} B^{\prime}\left(\lambda_{j}\right)^{1 / 2}\right.}{B_{j}}+c_{j}(B(\lambda))\right] \\
= & A^{\prime}(z)^{2} \sum_{j=1}^{n} \frac{\left(\lambda_{j}-A(0)\right)\left(\lambda_{j}-A(1)\right)}{(A(z)-A(0))(A(z)-A(1))\left(A(z)-\lambda_{j}\right)} \\
& \times\left[\frac{1}{2}\left(1-\nu_{j}^{-2}\right) \frac{1}{A(z)-\lambda_{j}}+c_{j}(B(\lambda)) B^{\prime}\left(\lambda_{j}\right)^{1 / 2} B^{\prime}(A(z))^{1 / 2}\right] .
\end{aligned}
$$

In order to obtain recursion relations, we examine the action of $A$ on $\{0,1, \infty\}$ and obtain

$$
\begin{gathered}
\frac{1}{2}\left(1-\nu_{j}^{-2}\right) \lambda_{j}+c_{j}\left(A_{2}(\lambda)\right)=\lambda_{j}^{2} c_{j}(\lambda) \\
\frac{1}{2}\left(1-\nu_{j}^{-2}\right)\left(\lambda_{j}-1\right)+c_{j}\left(A_{1}(\lambda)\right)=\left(\lambda_{j}-1\right)^{2} c_{j}(\lambda)
\end{gathered}
$$


(these last two relations are easily seen to be equivalent),

$$
\begin{aligned}
\frac{1}{2}\left(1-\nu_{j}^{-2}\right) \lambda_{j}-c_{j}\left(A_{3}(\lambda)\right) & =\lambda_{j}^{2} c_{j}(\lambda), \\
-c_{j}\left(A_{4}(\lambda)\right) & =c_{j}(\lambda), \\
\frac{1}{2}\left(1-\nu_{j}^{-2}\right)\left(\lambda_{j}-1\right)-c_{j}\left(A_{5}(\lambda)\right) & =\left(\lambda_{j}-1\right)^{2} c_{j}(\lambda),
\end{aligned}
$$

and using the motion $z \mapsto \bar{z}$, we obtain $c_{j}(\bar{\lambda})=\overline{c_{j}(\lambda)}$.

4.13. We consider an example. We let $\bar{S}_{\lambda}$ be the sphere with four distinguished points $\lambda, 0,1$, and $\infty$ and signature $(0,4, \mu, \nu, \nu, \nu)$. Let $\varphi_{\lambda}$ be the Fuchsian uniformizing connection for $\bar{S}_{\lambda}$ (actually, for $\nu=2=\mu$, we are considering the Euclidean uniformizing connection, about which much more is known). Using the relations derived in $\S 4.12$, we can compute the value $c(\lambda)=c_{1}(\lambda)$ for $\lambda$ a fixed point of one of the motions $A_{j}, j=1,2, \ldots, 5$. We have

$$
\begin{aligned}
& c\left(\frac{1}{2} \pm \frac{\sqrt{3}}{2} i\right)=\mp \frac{1}{2}\left(1-\mu^{-2}\right) \frac{\sqrt{3}}{3} i \\
& c(-1)=-\frac{1}{4}\left(1-\mu^{-2}\right), \quad c\left(\frac{1}{2}\right)=0
\end{aligned}
$$

and

$$
c(2)=\frac{1}{4}\left(1-\mu^{-2}\right) \text {. }
$$

(Note that these formulae are independent of $\nu$ and agree with our calculations in $\S 3.4$ for the Euclidean uniformization.)

Remark. The above results are closely related to Examples 2 and $5[\mathrm{Hm}, \mathrm{pp}$. 108 and 109]. It should also be noted that $\mathbf{C}-\{-1,0,1\}, \mathbf{C}-\{0,1,2\}$, and $\mathrm{C}-\left\{0, \frac{1}{2}, 1\right\}$ are affinely equivalent (for the cases with $\mu=\nu$ ), the appropriate maps being $z \mapsto z+1$ and $z \mapsto \frac{1}{2} z$. Further $z \mapsto z^{2}$ is a covering of $\mathbf{C}-\{0,1\}$ by $\mathbf{C}-\{-1,0,1\}$. These facts can be used to give alternate calculations for three of the accessory parameters listed above under some (obvious) restriction on the signature.

Assume next that $\nu>3$ (we can also handle the case $\nu=2=\mu$ ). Then we have seen that

$$
\lim _{\lambda \rightarrow 0} \varphi_{\lambda}=\Phi_{0}, \quad \lim _{\lambda \rightarrow 1} \varphi_{\lambda}=\Phi_{1}, \quad \lim _{\lambda \rightarrow \infty} \varphi_{\lambda}=\Phi_{\infty},
$$

where $\Phi_{j}$ is the Fuchsian uniformizing connection for $\widehat{\mathbf{C}}-\{0,1, \infty\}$ with signature

$$
\begin{array}{ll}
(0,3 ; \infty, \nu, \nu) & \text { for } j=0 \\
(0,3 ; \nu, \infty, \nu) & \text { for } j=1
\end{array}
$$

and

$$
(0,3 ; \nu, \nu, \infty) \text { for } j=\infty
$$

It follows that

$$
\lim _{\lambda \rightarrow 1} c(\lambda)=\infty \quad \text { if } \nu \neq \infty
$$


and

$$
\lim _{\lambda \rightarrow \infty} c(\lambda)=0
$$

as a matter of fact

$$
\lim _{\lambda \rightarrow 0} \lambda c(\lambda)=\frac{1}{2} \nu^{-2}, \quad \lim _{\lambda \rightarrow 1}(\lambda-1) c(\lambda)=\frac{1}{2} \nu^{-2}
$$

and

$$
\lim _{\lambda \rightarrow \infty} \lambda c(\lambda)=\frac{1}{2}\left(1-\mu^{-2}-\nu^{-2}\right)
$$

\section{FUNCTION GROUPS REPRESENTING SPHERES}

5.1. Let $\Gamma$ be a finitely generated nonelementary function group with invariant component $\Delta$. Assume that $\Gamma$ has type $(0, n+3), n \geq 1$. Let $T(\Gamma)$ be the Teichmüller space of deformations of $\Gamma$ supported on $\Delta$ (see, for example, [Kr3], where $\widehat{T}(\Gamma)$ is the symbol used for this space). We assume that $0,1, \infty$ are limit points of $\Gamma$. If $\mu \in M(\Gamma)$, the space of Beltrami coefficients for $\Gamma$ supported on $\Delta$, then $w^{\mu}$ denotes the unique normalized $\mu$-conformal automorphism of $\widehat{\mathbf{C}}$. As in $\S 4$, we let $S=\Delta / \Gamma, \pi: \Delta \rightarrow S$ and define $\nu \in M\left(S_{0}\right)$ by (4.2.3). We now define $\pi^{\mu}=w^{\nu} \circ \pi \circ\left(w^{\mu}\right)^{-1}$ and obtain in analogy to (4.2.4) the commutative diagram:

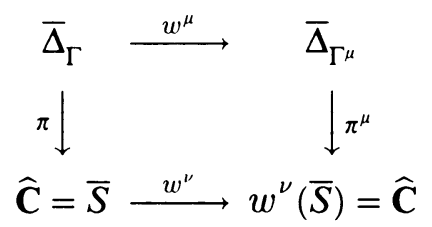

and a holomorphic covering map

$$
p: T(\Gamma) \rightarrow W^{n}
$$

given by the formulae

$$
\begin{aligned}
p([\mu]) & =\left(\pi^{\mu} \circ w^{\mu}\left(z_{1}\right), \ldots, \pi^{\mu} \circ w^{\mu}\left(z_{n}\right)\right) \\
& =\left(w^{\nu}\left(\lambda_{1}\right), \ldots, w^{\nu}\left(\lambda_{n}\right)\right), \quad \mu \in M(\Gamma) .
\end{aligned}
$$

Here $\left\{\lambda_{1}, \ldots, \lambda_{n}, 0,1, \infty\right\}=E=\bar{S}-S_{0}$, and $z_{1}, \ldots, z_{n+3} \in \bar{\Delta}_{\Gamma}$ are chosen so that $\pi\left(z_{j}\right)=\lambda_{j}, j=1, \ldots, n+3$.

We can now let $\varphi^{\mu}$ be the uniformizing connection for the Riemann surface $\bar{S}_{\nu}=w^{\nu}(\bar{S})$ (note that $E_{\nu}=\bar{S}_{\nu}-\left(S_{\nu}\right)_{0}=\left\{w^{\nu}\left(\lambda_{1}\right), \ldots, w^{\nu}\left(\lambda_{n}\right), 0,1, \infty\right\}$ ). In general, this connection depends on $\nu$ (or equivalently on $\mu$ ) complex analytically. Thus we have a meromorphic function $\varphi: T(\Gamma) \times \widehat{\mathbf{C}} \rightarrow \widehat{\mathbf{C}}$, whose restriction to $\{[\mu]\} \times \widehat{\mathbf{C}}$ is $\varphi^{\mu}$. Note that $\varphi^{\mu}$ depends on $[\mu]$ and not just on $w^{\nu}(\bar{S})$, because two different points on $T(\Gamma)$ representing a particular punctured sphere need not correspond to conjugate function groups. However, the difference between two uniformizing connections of the same surface is always a cusp form. This observation leads to a number of interesting questions that we hope to study in the future. 


\section{REFERENCES}

[B] L. Bers, On boundaries of Teichmüller spaces and on Kleinian groups. I, Ann. of Math. 91 (1970), 570-600.

[C] V. Chuckrow, On Schottky groups with applications to Kleinian groups, Ann. of Math. 88 (1968), 47-61.

[E] C. J. Earle, On quasiconformal extensions of Beurling-Ahlfors type, Contributions to Analysis, Academic Press, New York, 1974, pp. 107-124.

[EK] C. J. Earle and I. Kra, On sections of some holomorphic families of closed Riemann surfaces, Acta Math. 137 (1976), 49-79.

[G] R. C. Gunning, Lectures on Riemann surfaces, Math. Notes, Princeton Univ. Press, Princeton, N.J., 1966.

[He1] D. A. Hejhal, Universal covering maps for variable regions, Math. Z. 137 (1974), 7-20.

[He2] __, Monodromy groups and Poincaré series, Bull. Amer. Math. Soc. 84 (1978), 339-376.

[Hm] J. A. Hempel, On the uniformization of the n-punctured sphere, Bull. London Math Soc. 20 (1988), 97-115.

[Hi] E. Hille, Analytic function theory, Vol. II, Blaisdell, Waltham, Mass., 1962.

[J] T. Jørgensen, On discrete groups of Möbius transformations, Amer. J. Math. 98 (1976), 739749.

[KRV] L. Keen, H. Rauch, and A. T. Vasquez, Moduli of punctured tori and the accessory parameter of Lamé's equation, Trans. Amer. Math. Soc. 255 (1975), 201-230.

[Kr1] I. Kra, Deformations of Fuchsian gorups, Duke Math. J. 36 (1969), 537-546; 38 (1971), 499-508.

[Kr2] _ , Automorphic forms and Kleinian groups, Benjamin, Reading, Mass., 1972.

[Kr3] _ Canonical mappings between Teichmüller spaces, Bull. Amer. Math. Soc. 4 (1981), 143-179.

[Kr4] __ On the vanishing of and spanning sets for cusp forms, Acta Math. 153 (1984), 47-116.

[Mn] A. Marden, Universal properties of Fuchsian groups, Ann. of Math. Studies 79 (1974), 315339.

[Mt] B. Maskit, Parabolic elements in Kleinian groups, Ann. of Math. 117 (1983), 659-668.

[T] M. Tsuji, Modern potential theory in modern function theory, Chelsea, New York, 1975.

[W] C. K. Wong, A continuity theorem for Fuchsian groups, Trans. Amer. Math. Soc. 166 (1972), 225-239.

[ZT1] P. G. Zograf and L. A. Takhtadzhyan, An action for the Liouville equation as a generating function for accessory parameters and a potential for the Weil-Petersson metric on the Teichmüller space, Functional Anal. Appl. 19 (1985), 85-86.

[ZT2] __, On Liouville's equation, accessory parameters, and the geometry of the Teichmüller spaces for Riemann surfaces of genus 0, Math. USSR-Sb. 60 (1988), 143-161; transl. of Mat. Sb. 132 (1987), 147-166.

[ZT3] __ On uniformization of Riemann surfaces and the Weil-Petersson metric on Teichmüller and Schottky spaces, Math. USSR-Sb. 60 (1988), 297-313; transl. of Mat. Sb. 132 (1987), 304-321.

Department of Mathematics, State University of New York at Stony Brook, Stony BROOK, NEW YORK 11794 (Current address)

Mathematical Sciences Research Institute, Berkeley, California 94720 